\title{
The Poincaré-Hopf theorem for relative braid classes
}

\author{
Simone Munaò $^{1}$ - Robert Vandervorst ${ }^{1}$
}

Received: 3 February 2015 / Accepted: 28 November 2016 / Published online: 30 December 2016

(C) The Author(s) 2016. This article is published with open access at Springerlink.com

\begin{abstract}
This paper studies the existence and multiplicity of closed integral curves of smooth vector fields on the closed 2-disc $\mathbb{D}^{2}$ in the complement of a suitable geometric braid $y$-called a skeleton. The strands in a skeleton $y$ must be trajectories of the vector field and the 1-periodic orbits to be counted must also be representatives of a suitable relative braid class $x$ in the complement of $y$. From Leray-Schauder degree theory a signed count of the closed trajectories representing $x$ in the complement of $y$ is independent of the vector field. It therefore suffices to compute, or estimate the signed count of periodic orbits for a particularly convenient vector field. We show that the signed count equals the Euler-Floer characteristic of Braid Floer homology, (cf. van den Berg et al. in J Differ Equ 259(5):1663-1721, 2015). The latter can be computed via a finite cube complex which serves as a model for the given braid class.
\end{abstract}

\section{Introduction}

Let $\mathbb{D}^{2} \subset \mathbb{R}^{2}$ denote the standard (closed) 2-disc in the plane with coordinates $x=(p, q)$ and let $X(x, t)$ be a smooth 1-periodic vector field on $\mathbb{D}^{2}$. To be more precise,

(i) $X \in C^{\infty}\left(\mathbb{D}^{2} \times \mathbb{R} / \mathbb{Z} ; \mathbb{R}^{2}\right)$, i.e. $X(x, t+1)=X(x, t)$ for all $x \in \mathbb{D}^{2}$ and $t \in \mathbb{R}$;

(ii) $X(x, t) \cdot v=0$ for all $x \in \partial \mathbb{D}^{2}$, where $\nu$ the outward unit normal on $\partial \mathbb{D}^{2}$.

The set of vector fields satisfying Hypotheses (i)-(ii) is denoted by $\mathscr{X}\left(\mathbb{D}^{2} \times \mathbb{R} / \mathbb{Z}\right)$. Closed integral curves $x(t)$ of $X$ are integral curves ${ }^{1}$ of $X$ for which $x(t+\ell)=x(t)$ for some $\ell \in \mathbb{N}$. Every integral curve of $X$ with minimal period $\ell$ may be regarded as a geometric braid with

\footnotetext{
${ }^{1}$ Integral curves of $X$ are smooth functions $x: \mathbb{R} \rightarrow \mathbb{D}^{2} \subset \mathbb{R}^{2}$ that satisfy the differential equation $x_{t}=X(x, t)$.

$凶$ Robert Vandervorst

r.c.a.m.vander.vorst@vu.nl

Simone Munaò

s.munao@vu.nl

1 Department of Mathematics, Vrije Universiteit Amsterdam, Amsterdam, The Netherlands
} 

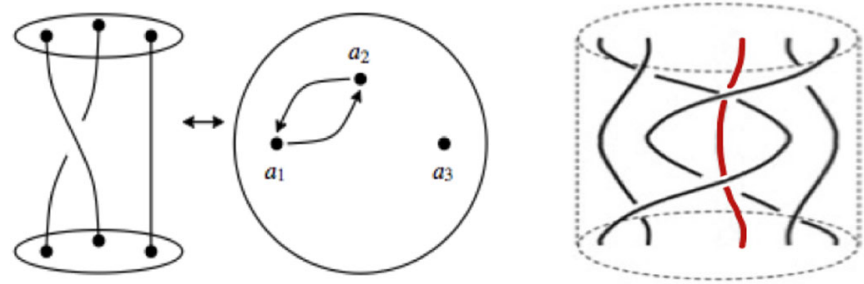

Fig. 1 A 2-periodic and 1-periodic closed integral curve represented as 3-strand braids (left). A closed integral curve with minimal period $\ell$ yields a $\ell$-periodic point of the associated time- 1 map (middle). A relative braid with the skeleton (black) and one free strand (red) (right) (color figure online)

$\ell$ strands by considering all translates $x^{i}(t)=x(t+i)$ for $i=1, \ldots, \ell$ and $t \in[0,1]$, see Fig. 1 (left). They indeed form a geometric braid since the strands $x^{i}$ are distinct integral curves of $X$ and cannot intersect therefore! Multiple closed integral curves of various periods yield a multi-strand geometric braid.

Let $y$ be a geometric braid consisting of closed integral curves of $X$. We will refer to $y$ as a skeleton for $X$. The strands $y^{i}(t), i=1, \ldots, m$ satisfy the periodicity condition $y(0)=y(1)$ as point sets, i.e. $y^{i}(0)=y^{\sigma(i)}(1)$ for some permutation $\sigma \in S_{m}$. Let $x=\left\{x^{1}(t), \ldots x^{n}(t)\right\}$ be a geometric braid in the complement of $y$, i.e. the 'union' $x$ rel $y:=\left\{x^{1}(t), \ldots x^{n}(t), y^{1}(t), \ldots, y^{m}(t)\right\}$ is again a geometric braid-the strands in $x$ do not intersect the strands in $y$. The pair $x$ rel $y$ is called a relative braid, or 2-colored braid. Two relative braids $x$ rel $y$ and $x^{\prime}$ rel $y^{\prime}$ are equivalent if there exists a homotopy of relative braids connecting $x$ rel $y$ to $x^{\prime}$ rel $y^{\prime}$. The equivalence class is denoted by $[x$ rel $y]$ and is called a relative braid class. The set of relative braids $x^{\prime}$ rel $y^{\prime} \in[x$ rel $y]$, keeping $y^{\prime}$ fixed, is denoted by $\left[x^{\prime}\right]$ rel $y^{\prime}$ and is called relative braid class fiber over $y^{\prime}$.

A relative braid class $\left[x\right.$ rel $y$ ] is proper if components $x_{c} \subset x$ cannot be deformed onto (i) the boundary $\partial \mathbb{D}^{2}$, (ii) itself, ${ }^{2}$ or other components $x_{c}^{\prime} \subset x$, or (iii) components in $y_{c} \subset y$, see [17] for details.

The central question is: given a skeleton $y$ of integral curves of $X$, do there exist integral curves $x$ in the complement of $y$ ? In this paper we are concerned with relative braids $x$ rel $y$ for which $x$ has only one strand, i.e. $x$ is 1-periodic $-x(t+1)=x(t)$.

\subsection{A summary of braid Floer homology}

Fix a Hamiltonian vector field $X_{H}$ in $\mathscr{X}\left(\mathbb{D}^{2} \times \mathbb{R} / \mathbb{Z}\right)$ of the form

$$
X_{H}(x, t)=J \nabla H(x, t), \quad J=\left(\begin{array}{rr}
0 & -1 \\
1 & 0
\end{array}\right),
$$

where $H$ is a Hamiltonian function with the properties:

(i) $H \in C^{\infty}\left(\mathbb{D}^{2} \times \mathbb{R} / \mathbb{Z} ; \mathbb{R}\right)$, i.e. $H(x, t+1)=H(x, t)$ for all $x \in \mathbb{D}^{2}$ and $t \in \mathbb{R}$;

(ii) $\left.H(x, t)\right|_{x \in \partial \mathbb{D}^{2}}=0$, for all $t \in \mathbb{R}$.

For 1-periodic closed integral curves of $X_{H}$ the the Hamilton action is given by

$$
\mathscr{A}_{H}(x)=\int_{0}^{1} \frac{1}{2} J x \cdot x_{t}-H(x, t) d t,
$$

2 This condition is separated into two cases: (a) a component in $x$ cannot be deformed into a single strand, or (b) if a component in $x$ can be deformed into a single strand, then the latter necessarily intersects $y$ or a different component in $x$. 
Critical points of the action functional $\mathscr{A}_{H}$ are in one-to-one correspondence with 1-periodic closed integral curves of $X_{H}$. Assume that $y=\left\{y^{j}(t)\right\}$ is a skeleton for the Hamilton vector field $X_{H}$. Consider 1-periodic closed integral curves of $X_{H}$ in the complement of $y$ by specifying a proper relative braid class $\left[x\right.$ rel $y$ ]. The set of critical point of $\mathscr{A}_{H}$ in the complement of $y$ which lie in the relative braid class fiber $[x]$ rel $y$ is denoted by Crit $\mathscr{A}_{H}([x]$ rel $y)$. In order to understand the set Crit $\mathscr{A}_{H}([x] \mathrm{rel} y)$ we consider the negative $L^{2}$-gradient flow of $\mathscr{A}_{H}$, which yields the Cauchy-Riemann equations

$$
u_{s}(s, t)-J u_{t}(s, t)-\nabla H(u(s, t), t)=0 .
$$

The stationary solutions of (3) are the critical points of $\mathscr{A}_{H}$.

To a braid $y$ one can assign an integer Cross $(y)$ which counts the number of crossings (with sign) of strands in the standard planar projection of $y$. In the case of a relative braid $x$ rel $y$ the number Cross $(x$ rel $y)$ is an invariant of the relative braid class [ $x$ rel $y$ ]. In [17] a monotonicity lemma is proven, which states that along solutions $u(s, t)$ of the nonlinear Cauchy-Riemann equations, the number $\operatorname{Cross}(u(s, \cdot)$ rel $y$ ) is non-increasing (the jumps correspond to 'singular braids', i.e. 'braids' for which intersections occur). As a consequence an isolation property for proper relative braid classes exists: the bounded solutions $u(s, t)$ of the Cauchy-Riemann equations for which $u$ rel $y$ lies in a proper relative braid class fiber $[x]$ rel $y$, form the set $\mathscr{M}([x]$ rel $y ; H)$, which is compact and isolated with respect to the topology of uniform convergence on compact subsets of $\mathbb{R}^{2}$. These facts provide all the ingredients to use Floer's approach towards Morse Theory for the Hamilton action, cf. [8]. For generic Hamiltonians which satisfy (i) and (ii) above and for which $y$ is a skeleton, the critical points in Crit $\mathscr{A}_{H}([x]$ rel $y)$ of the action $\mathscr{A}_{H}$ are non-degenerate and the sets of connecting orbits $\mathscr{M}_{x_{-}, x_{+}}([x] \mathrm{rel} y ; H)$ are smooth finite dimensional manifolds with

$$
\operatorname{dim} \mathscr{M}_{x_{-}, x_{+}}([x] \operatorname{rel} y ; H)=\mu^{C Z}\left(x_{-}\right)-\mu^{C Z}\left(x_{+}\right),
$$

where $\mu^{C Z}\left(x_{ \pm}\right)$are the Conley-Zehnder indices of $x_{ \pm} \in \mathrm{Crit}_{\mathscr{A}_{H}}([x]$ rel $y)$, cf. Eqs. (15) and (21).

Define the free abelian groups $C_{k}$ over the critical points of index $k$, with coefficients in $\mathbb{Z}_{2}$, i.e.

$$
C_{k}([x] \operatorname{rel} y ; H):=\bigoplus_{\substack{x_{0} \in \operatorname{Crit}_{\mathscr{A}_{H}}([x] \text { rel } y), \mu^{C Z}\left(x_{0}\right)=k}} \mathbb{Z}_{2}\left\langle x_{0}\right\rangle,
$$

and the boundary operator

$$
\partial_{k}=\partial_{k}([x] \operatorname{rel} y ; H): C_{k} \rightarrow C_{k-1},
$$

which counts the number of orbits (modulo 2) between critical points of index $k$ and $k-1$ respectively. Analysis of the spaces $\mathscr{M}_{x_{-}, x_{+}}([x] \mathrm{rel} y ; H)$ reveals that $\left(C_{*}, \partial_{*}\right)$ is a chain complex and its (Floer) homology is denoted by $\mathrm{HB}_{*}([x]$ rel $y ; H)$. Different choices of $H$ and different fibers yield isomorphic Floer homologies and

$$
\mathrm{HB}_{*}([x \text { rel } y]):=\lim _{\leftarrow} \mathrm{HB}_{*}([x] \operatorname{rel} y ; H),
$$

where the inverse limit is defined with respect to the canonical isomorphisms $f_{k}$ : $\operatorname{HB}_{k}([x] \operatorname{rel} y, H) \rightarrow \operatorname{HB}_{k}\left(\left[x^{\prime}\right] \operatorname{rel} y^{\prime}, H^{\prime}\right)$. Some properties are:

(a) the groups $\operatorname{HB}_{k}([x$ rel $y])$ are defined for all $k \in \mathbb{Z}$ and are of finite rank;

(b) $\mathrm{HB}_{k}([x] \operatorname{rel} y)=\lim \mathrm{HB}_{*}([x] \operatorname{rel} y ; H)$ are invariants for the fibers in the same relative braid class $[x$ rel $y]$, i.e. if $x$ rel $y \sim x^{\prime} \operatorname{rel} y^{\prime}$, then $\operatorname{HB}_{k}([x] \operatorname{rel} y) \cong \operatorname{HB}_{k}\left(\left[x^{\prime}\right] \operatorname{rel} y^{\prime}\right)$; 
(c) if $(x$ rel $y) \cdot \Delta^{2 \ell}$ denotes composition with $\ell$ full twists, then $\operatorname{HB}_{k}\left(\left[(x\right.\right.$ rel $\left.\left.y) \cdot \Delta^{2 \ell}\right]\right) \cong$ $\mathrm{HB}_{k-2 \ell}([x$ rel $y])$ for all $k \in \mathbb{Z}$.

In [17] Braid Floer homology is used as a Morse type theory for closed integral curves of $X_{H}$ forced by a skeleton $y$. For generic Hamiltonians define the Poincaré polynomial

$$
P_{\tau}\left(\operatorname{Crit}_{\mathscr{A}_{H}}([x] \operatorname{rel} y)\right):=\sum_{k \in \mathbb{Z}} c_{k}([x] \operatorname{rel} y ; H) \tau^{k},
$$

where $c_{k}=\operatorname{dim} C_{k}([x] \operatorname{rel} y ; H)$. Then,

$$
P_{\tau}\left(\operatorname{Crit}_{\mathscr{A}_{H}}([x] \operatorname{rel} y)\right)=P_{\tau}([x \operatorname{rel} y])+(1+\tau) Q_{\tau},
$$

where $P_{\tau}([x$ rel $y])$ is the Poincaré polynomial of $\mathrm{HB}_{*}([x$ rel $y])$ and $Q_{\tau} \geq 0$, cf. [17, Thm. 11.3]. Braid Floer homology cannot be applied to arbitrary vector fields $X$. The objective of this paper to extract an invariant from $\mathrm{HB}_{*}([x$ rel $y])$ which provides a forcing theory for arbitrary vector fields $X$.

\subsection{The Euler-Floer characteristic and the Poincaré-Hopf index formula}

A 1-periodic function $x \in H^{1}(\mathbb{R} / \mathbb{Z}){ }^{3}$ with $|x(t)| \leq 1$, is an isolated closed integral curve of $X$ if there exists an $\epsilon>0$ such that $x$ is the only solution of the differential equation

$$
\mathscr{E}(x)(t)=\frac{d x(t)}{d t}-X(x(t), t)=0,
$$

in a ball $B_{\epsilon}(x)$ in $H^{1}(\mathbb{R} / \mathbb{Z})$. A closed integral curve is non-degenerate if the linearized operator $D_{x} \mathscr{E}(x):=\frac{d}{d t}-D_{x} X(x(t), t): H^{1}(\mathbb{R} / \mathbb{Z}) \rightarrow L^{2}(\mathbb{R} / \mathbb{Z})$ is invertible. By standard regularity arguments closed integral curves are smooth functions $x \in C^{\infty}(\mathbb{R} / \mathbb{Z})$.

For non-degenerate 1-periodic closed integral curves we define an index as follows. Consider the initial value problem

$$
\left\{\begin{array}{l}
\frac{d \Psi}{d t}-D_{x} X(x(t), t) \Psi=0 \\
\Psi(0)=\mathrm{Id}
\end{array}\right.
$$

Since the non-degeneracy of $x$ implies that $1 \notin \sigma(\Psi(1))$ (cf. Lemma 3 ) we define the index of a non-degenerate 1-periodic closed integral curve by

$$
\iota(x):=-\operatorname{sgn}(\operatorname{det}(\Psi(1)-\mathrm{Id})) \in\{-1,1\} .
$$

In Sect. 2 we provide a topological definition of the index which allows an extension of the index to isolated closed integral curves, cf. Sect. 5.2.

Theorem 1 (Poincaré-Hopf index formula) Let y be a skeleton of closed integral curves of a vector field $X \in \mathscr{X}\left(\mathbb{D}^{2} \times \mathbb{R} / \mathbb{Z}\right)$ and let $[x$ rel $y]$ be a proper relative braid class. Suppose that all 1-periodic closed integral curves $x_{0}$ of $X$, for which $x_{0}$ rel $y$ lies in the fiber $[x]$ rel $y$, are isolated. Then,

$$
\sum_{x_{0}} \mathrm{l}\left(x_{0}\right)=\chi(x \operatorname{rel} y)
$$

3 By $H^{1}(\mathbb{R} / \mathbb{Z})$ we denote the Sobolev space of $\mathbb{R}^{2}$-valued $L^{2}$-functions on $\mathbb{R} / \mathbb{Z}$ whose weak derivatives are contained in $L^{2}$. 

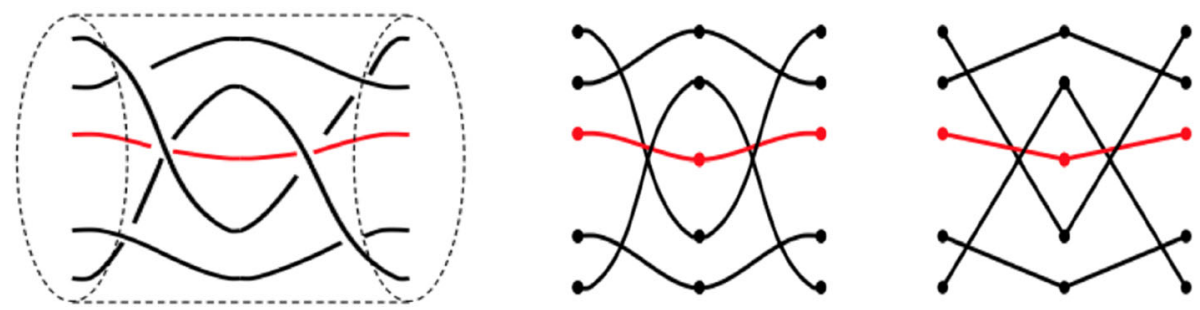

Fig. 2 A positive relative braid (left), anchor points (middle), and piecewise linear discretization (right)

where

$$
\chi(x \text { rel } y)=\sum_{k \in \mathbb{Z}}(-1)^{k} \operatorname{dim} \operatorname{HB}_{k}([x \text { rel } y])
$$

is the Euler-Floer characteristic of $\mathrm{HB}_{*}([x$ rel $y])$.

The index formula can be used to obtain existence results for closed integral curves in proper relative braid classes.

Corollary 1 Let y be a skeleton of closed integral curves of a vector field $X \in \mathscr{X}\left(\mathbb{D}^{2} \times \mathbb{R} / \mathbb{Z}\right)$ and let $[x$ rel $y]$ be a proper relative braid class. If $\chi(x$ rel $y) \neq 0$, then there exist closed integral curves $x_{0}$ such that $x_{0}$ rel $y$ lies in the fiber $[x]$ rel $y$.

Remark 1 In this paper we do not address the question whether the closed integral curves are non-constant. However, closed integral curves in different relative braid classes correspond to different periodic points! By considering relative braid classes where $x$ consists of more than one strand one can study non-constant closed integral curves. Braid Floer homology for relative braids with $x$ consisting of $n$ strands is defined in [17]. The ideas in this paper extend to relative braid classes with multi-strand braids $x$.

\subsection{Discretization and computability}

The second part of the paper deals with the computability of the Euler-Floer characteristic. The latter is carried out in three steps:

(a) compose $x$ rel $y$ with $\ell \geq 0$ full twists $\Delta^{2}$, such that $(x \operatorname{rel} y) \cdot \Delta^{2 \ell}$ is isotopic to a positive braid $x^{+}$rel $y^{+}$- a braid with only positive crossings;

(b) relative braids $x^{+}$rel $y^{+}$are isotopic to Legendrian braids $x_{L}$ rel $y_{L}$ on $\mathbb{R}^{2}$, i.e. braids which have the form $x_{L}=\left(q_{t}, q\right)$ and $y_{L}=\left(Q_{t}, Q\right)$, where $q=\pi_{2} x$ and $Q=\pi_{2} y$, and $\pi_{2}$ is the projection onto the $q$-coordinate, and both $q$ and $Q$ take values in [-1.1];

(c) discretize $q$ and $Q=\left\{Q^{j}\right\}$ to $q_{d}=\left\{q_{i}\right\}$, with $q_{i}=q(i / d), i=0, \ldots, d$ and $Q_{D}=$ $\left\{Q_{D}^{j}\right\}$, with $Q_{D}^{j}=\left\{Q_{i}^{j}\right\}$ and $Q_{i}^{j}=Q^{j}(i / d)$ respectively, and consider the piecewise linear interpolations connecting the anchor points $q_{i}$ and $Q_{i}^{j}$ for $i=0, \ldots, d$, see Fig. 2. A discretization $q_{D}$ rel $Q_{D}$ is admissible if the linear interpolation is isotopic to $q$ rel $Q$. All such discretizations form the discrete relative braid class $\left[q_{D}\right.$ rel $\left.Q_{D}\right]$, for which each fiber is a finite cube complex, cf. [9]. 

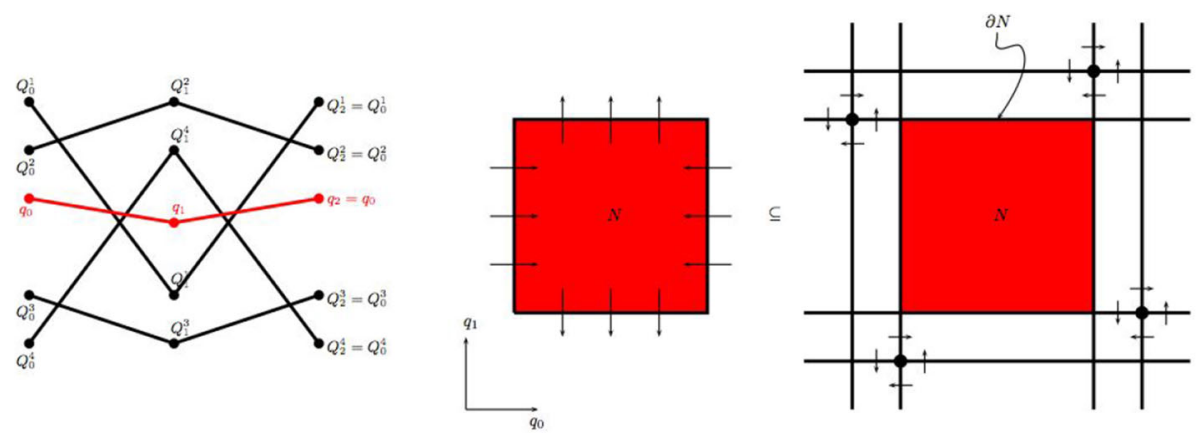

Fig. 3 The relative braid fiber $\left[q_{D}\right] \operatorname{rel} Q_{D}$ and $N=\operatorname{cl}\left(\left[q_{D}\right] \operatorname{rel} Q_{D}\right)$

Remark 2 If the number of discretization points is not large enough, then the discretizations may not be admissible and therefore do not capture the topology of the braid class. See [9] and Sect. 9.1 for more details.

For $d>0$ large enough there exists an admissible discretization $q_{D}$ rel $Q_{D}$ for any Legendrian representative $x_{L}$ rel $y_{L} \in[x$ rel $y]$ and thus an associated discrete relative braid class $\left[q_{D}\right.$ rel $\left.Q_{D}\right]$. In [9] an invariant for discrete braid classes was introduced. Let $\left[q_{D}\right] \operatorname{rel} Q_{D}$ denote a fiber in $\left[q_{D}\right.$ rel $\left.Q_{D}\right]$, which is a cube complex with a finite number of connected components and their closures are denoted by $N_{j}$. The faces of the hypercubes in $N_{j}$ that compose the boundary $\partial N_{j}$ can be co-oriented in the direction of decreasing the number of crossings in $q_{D}$ rel $Q_{D}$. We define $N_{j}^{-}$as the closure of the subset of the boundary defined by the faces with outward pointing co-orientation. Figure 3 below explains the sets $N_{j}$ and $N_{j}^{-}$for the example in Fig. 2 . The sets $N_{j}^{-}$are called exit sets.

The invariant for a fiber is given by the Conley index

$$
\mathrm{HC}_{*}\left(\left[q_{D}\right] \operatorname{rel} Q_{D}\right)=\bigoplus_{j} H_{*}\left(N_{j}, N_{j}^{-}\right) .
$$

This discrete braid invariant is well-defined for any $d>0$ for which there exist admissible discretizations and is independent of both the particular fiber and the discretization size $d$. For the associated Euler characteristic we therefore write $\chi\left(q_{D}\right.$ rel $\left.Q_{D}\right)$.

Theorem 2 Let $[x$ rel $y$ ] be a proper relative braid class and let $\ell \geq 0$ be an integer such that $(x \operatorname{rel} y) \cdot \Delta^{2 \ell}$ is isotopic to a positive braid $x^{+} \operatorname{rel} y^{+}$. Suppose $q_{D} \operatorname{rel} Q_{D}$ is an admissible discretization, for some $d>0$, of a Legendrian representative $x_{L}$ rel $y_{L} \in\left[x^{+} \operatorname{rel} y^{+}\right]$. Then,

$$
\chi(x \operatorname{rel} y)=\chi\left(q_{D} \operatorname{rel} Q_{D}^{*}\right),
$$

where $Q_{D}^{*}$ is an augmentation of $Q_{D}$ by adding the constant strands \pm 1 to $Q_{D}$.

Remark 3 If we combine Theorems 1 and 2 we obtain a direct relation between 1-periodic closed integral curves in a braid class fiber $[x]$ rel $y$ and the Euler characteristic of an appropriately constructed discretized model of the braid class fiber. Using the methods in this paper this relation can be proved directly without using Braid Floer homology. The advantage of the approach taken in this paper is that we also relate the discretized models to Braid Floer homology. 


\section{An index for loops of $2 \times 2$ matrices}

In the introduction we defined an index for non-degenerate closed integral curves of a vector field $X$, cf. Eq. (6). In this section we will give a detailed account of the index based on the theory of parity and degree theory, cf. [5].

Let $t \mapsto A(t) \in \mathrm{M}_{2 \times 2}(\mathbb{R}), t \in \mathbb{R} / \mathbb{Z}$ be a smooth loop of $2 \times 2$ matrices, which we denote by $A$. The linear operator

$$
\mathcal{L}_{A}:=\frac{d}{d t}-A(t): H^{1}(\mathbb{R} / \mathbb{Z}) \rightarrow L^{2}(\mathbb{R} / \mathbb{Z}),
$$

is a Fredholm operator of index 0 . If $\mathcal{L}_{A}$ is invertible then $A$ is called a non-degenerate loop. To a non-degenerate loop $A$ we assign an index by considering paths $\eta \mapsto \mathcal{F}_{\Theta}(\eta), \eta \in I=[0,1]$, of the form $\mathcal{F}_{\Theta}(\eta)=\frac{d}{d t}-\mathcal{R}(t ; \eta)$, where $\mathcal{R}(t ; 0)=\Theta \in \mathrm{M}_{2 \times 2}(\mathbb{R})$, with $\sigma(\Theta) \cap 2 \pi i \mathbb{Z}=\varnothing$ and $\mathcal{R}(t ; 1)=A(t)$. By construction $\eta \mapsto \mathcal{F}_{\Theta}(\eta)$ is a path of linear Fredholm operators on index 0 with invertible end points. Notation: $\mathcal{F}_{\Theta}: I \rightarrow \operatorname{Fred}_{0}\left(H^{1}, L^{2}\right)$, $\operatorname{where} \operatorname{Fred}_{0}\left(H^{1}, L^{2}\right)$ denotes the space of index 0 Fredholm operators from $H^{1}(\mathbb{R} / \mathbb{Z})$ to $L^{2}(\mathbb{R} / \mathbb{Z})$.

Following [5, §3] we denote by $\Sigma \subset \operatorname{Fred}_{0}\left(H^{1}, L^{2}\right)$ the set of non-invertible operators in $\operatorname{Fred}_{0}\left(H^{1}, L^{2}\right)$ and by $\Sigma_{1} \subset \Sigma$ the non-invertible operators with a 1-dimensional kernel. Since the end points of $\mathcal{F}_{\Theta}$ are invertible one can choose the path $\eta \mapsto \mathcal{R}(t ; \eta)$ in such a way that $\mathcal{F}_{\Theta}(\eta)$ intersects $\Sigma$ in $\Sigma_{1}$ and all intersections are transverse, cf. [5, Thm. 3.12]. Such paths are called generic paths. The number of transverse intersections with $\Sigma_{1}$ is finite and is denoted by $\gamma$. The integer $(-1)^{\gamma}$ is called the parity of the path $\eta \mapsto \mathcal{F}_{\Theta}(\eta), \eta \in I$, and is denoted parity $\left(\mathcal{F}_{\Theta}(\eta), I\right)$, cf. [5].

Definition 1 Let $A$ be a non-degenerate loop and let $\Theta \in \mathrm{M}_{2 \times 2}(\mathbb{R})$ with $\sigma(\Theta) \cap 2 \pi i \mathbb{Z}=\varnothing$. The index of a non-degenerate loop $A$ of matrices is defined as

$$
\iota(A):=\operatorname{sgn}(\operatorname{det}(\Theta))(-1)^{\gamma+1},
$$

where $\gamma=\#$ intersections of the (generic) path $\mathcal{F}_{\Theta}(\eta)$ with $\Sigma_{1}$.

It remains to show that the index $\iota(A)$ is well-defined. To do so we use an alternative formulation in terms of the Leray-Schauder degree, cf. [5]. Choose a path $\eta \mapsto \mathcal{M}(\eta)$, with $\mathcal{M}(\eta): L^{2}(\mathbb{R} / \mathbb{Z}) \rightarrow H^{1}(\mathbb{R} / \mathbb{Z})$ invertible all $\eta \in I$, such that $\mathcal{D}_{\mathcal{M}, \Theta}(\eta):=\mathcal{M}(\eta) \mathcal{F}_{\Theta}(\eta)=$ $\operatorname{Id}-K_{\Theta}(\eta): H^{1}\left(\mathbb{R} / \mathbb{Z} ; \mathbb{R}^{2}\right) \rightarrow H^{1}(\mathbb{R} / \mathbb{Z})$, with $K_{\Theta}(\eta)$ compact. Such a path $\eta \mapsto \mathcal{M}(\eta)$ always exists and is called a parametrix for $\mathcal{F}_{\Theta}(\eta)$, cf. [3, Prop. 2.3] and [4, Thm. 1.3.6]. The Leray-Schauder degree of an invertible linear operator $\operatorname{Id}-K$ is given by $\operatorname{deg}_{L S}(\operatorname{Id}-K)=$ $(-1)^{\beta}$, where

$$
\beta=\sum_{\sigma_{j}>1, \sigma_{j} \in \sigma(K)} \beta_{j}, \quad \beta_{j}=\operatorname{dim}\left(\bigcup_{i=1}^{\infty} \operatorname{ker}\left(\sigma_{j} \mathrm{Id}-K\right)^{i}\right),
$$

which will be referred to as the Morse index of Id $-K$, cf. [10]. The partity of a path $\mathcal{D}_{\mathcal{M}, \Theta}(\eta)$ is defined by parity $\left(\mathcal{D}_{\mathcal{M}, \Theta}(\eta), I\right)=\operatorname{deg}_{L S}\left(\mathcal{D}_{\mathcal{M}, \Theta}(0)\right) \cdot \operatorname{deg}_{L S}\left(\mathcal{D}_{\mathcal{M}, \Theta}(1)\right)$, cf. [5, Thm 3.1.2] and is independent of the chosen parametrix. Thus for $\mathcal{F}_{\Theta}(\eta)$ we define $\operatorname{parity}\left(\mathcal{F}_{\Theta}(\eta), I\right):=\operatorname{parity}\left(\mathcal{D}_{\mathcal{M}, \Theta}(\eta), I\right)$, For generic paths $\mathcal{F}_{\Theta}(\eta)$ parity is given by the above intersection formula. For a list of properties of parity see [4-7].

Lemma $1 \mathrm{\iota}(A)=-\operatorname{sgn}(\operatorname{det}(\Theta))(-1)^{\beta_{\mathcal{M}}(\Theta)} \operatorname{deg}_{L S}\left(\mathcal{D}_{\mathcal{M}, \Theta}(1)\right)$, where $\beta_{\mathcal{M}}(\Theta)$ is the Morse index of $\mathcal{D}_{\mathcal{M}, \Theta}(0)$. 
Proof From the Leray-Schauder degree characterization of parity we have we have:

$$
\begin{aligned}
(-1)^{\gamma} & =\operatorname{parity}\left(\mathcal{F}_{\Theta}(\eta), I\right)=\operatorname{parity}\left(\mathcal{D}_{\mathcal{M}, \Theta}(\eta), I\right) \\
& =\operatorname{deg}_{L S}\left(\mathcal{D}_{\mathcal{M}, \Theta}(0)\right) \cdot \operatorname{deg}_{L S}\left(\mathcal{D}_{\mathcal{M}, \Theta}(1)\right)=(-1)^{\beta_{\mathcal{M}}(\Theta)} \operatorname{deg}_{L S}\left(\mathcal{D}_{\mathcal{M}, \Theta}(1)\right),
\end{aligned}
$$

cf. [4,5], which proves that the characterization $\mathrm{\iota}(A)$ does not depend on $\mathcal{N}(\eta)$.

Lemma 2 The index $\mathrm{L}(A)$ is independent of the choice of $\Theta \in \mathrm{M}_{2 \times 2}(\mathbb{R})$.

Proof Let $\Theta$ and $\Theta^{\prime}$ be admissible matrices and let $\eta \mapsto \mathcal{G}(\eta)$ be a path connecting $\mathcal{G}(0)=$ $\frac{d}{d t}-\Theta$ and $\mathcal{G}(1)=\frac{d}{d t}-\Theta^{\prime}$. For the parities it holds that

$$
\operatorname{parity}\left(\mathcal{F}_{\Theta}(\eta), I\right)=\operatorname{parity}(\mathcal{G}(\eta), I) \cdot \operatorname{parity}\left(\mathcal{F}_{\Theta^{\prime}}(\eta), I\right) .
$$

To compute parity $(\mathcal{G}(\eta), I)$ we consider a special parametrix $\mathcal{M}=\left(\frac{d}{d t}+\mu\right)^{-1}, \mu>0$. From the definition of parity we have that

$$
\operatorname{parity}(\mathcal{G}(\eta), I)=\operatorname{parity}(\mathcal{M} \mathcal{G}(\eta), I)=\operatorname{deg}_{L S}(\mathcal{M} \mathcal{G}(0)) \cdot \operatorname{deg}_{L S}(\mathcal{M} \mathcal{G}(1)) \text {. }
$$

We now compute the Leray-Schauder degrees of $\mathcal{M G}(0)$ and $\mathcal{M} \mathcal{G}(1)$. We start with $\Theta$ and in order to compute the degree we determine the Morse index. Consider the eigenvalue problem

$$
\mathcal{M G}(0) \psi=\lambda \psi, \quad \lambda \in \mathbb{R},
$$

which is equivalent to $(1-\lambda) \frac{d \psi}{d t}=(\Theta+\lambda \mu) \psi$. Non-trivial solutions are given by $\psi(t)=$ $\exp \left(\frac{\Theta+\lambda \mu}{1-\lambda} t\right) \psi_{0}$, which yields the condition $\frac{\theta+\lambda \mu}{1-\lambda}=2 \pi k i, k \in \mathbb{Z}$, where $\theta$ is an eigenvalues of $\Theta$. We now consider three cases:

(i) $\theta_{ \pm}=a \pm i b$. In case of a negative eigenvalue $\lambda$ we have $\frac{a+\lambda \mu}{1-\lambda}=0$ and $\frac{b}{1-\lambda}=2 \pi k$. The same $\lambda<0$ also suffices for the conjugate eigenvalue via $\frac{-b}{1-\lambda}=-2 \pi k$. This implies that any eigenvalue $\lambda<0$ has multiplicity 2 , and thus $\operatorname{deg}_{L S}(\mathcal{M} \mathcal{G}(0))=1$.

(ii) $\theta_{ \pm} \in \mathbb{R}, \theta_{-} \cdot \theta_{+}>0$. In case of a negative eigenvalue $\lambda$ we have $\frac{\theta_{ \pm}+\lambda \mu}{1-\lambda}=0$ and thus $\lambda_{ \pm}=-\frac{\theta_{ \pm}}{\mu}$, which yields two negative or two positive eigenvalues. As before $\operatorname{deg}_{L S}(\mathcal{M G}(0))=1$.

(iii) $\theta_{ \pm} \in \mathbb{R}, \theta_{-} \cdot \theta_{+}<0$. From case (ii) we easily derive that there exist two eigenvalues $\lambda_{ \pm}$, one positive and one negative, and therefore $\operatorname{deg}_{L S}(\mathcal{M} \mathcal{G}(0))=-1$.

These cases combined imply that $\operatorname{deg}_{L S}(\mathcal{M} \mathcal{G}(0))=\operatorname{sgn}(\operatorname{det}(\Theta))$ and thus

$$
\operatorname{parity}(\mathcal{G}(\eta), I)=\operatorname{sgn}(\operatorname{det}(\Theta)) \cdot \operatorname{sgn}\left(\operatorname{det}\left(\Theta^{\prime}\right)\right) \text {. }
$$

From the latter we derive:

$$
\begin{aligned}
\operatorname{sgn}(\operatorname{det}(\Theta)) & \cdot \operatorname{parity}\left(\mathcal{F}_{\Theta}(\eta), I\right) \\
& =\operatorname{sgn}(\operatorname{det}(\Theta)) \cdot \operatorname{sgn}(\operatorname{det}(\Theta)) \cdot \operatorname{sgn}\left(\operatorname{det}\left(\Theta^{\prime}\right)\right) \cdot \operatorname{parity}\left(\mathcal{F}_{\Theta^{\prime}}(\eta), I\right) \\
& =\operatorname{sgn}\left(\operatorname{det}\left(\Theta^{\prime}\right)\right) \cdot \operatorname{parity}\left(\mathcal{F}_{\Theta^{\prime}}(\eta), I\right),
\end{aligned}
$$

which proves the independence of $\Theta$.

\section{An analytic characterization and the Conley-Zehnder index}

From the intersection number definition of the index $\mathrm{I}(A)$ in Definition 1 a different characterization based on spectral properties of the operator $\mathcal{L}_{A}$ can be derived. Consider the initial value problem $\mathcal{L}_{A} \Psi_{A}=\frac{d \Psi_{A}}{d t}-A(t) \Psi_{A}=0$, with $\Psi_{A}(0)=$ Id. 
Lemma 3 The following criteria for non-degeneracy are equivalent:

(i) the operator $\mathcal{L}_{A}: H^{1}(\mathbb{R} / \mathbb{Z}) \rightarrow L^{2}(\mathbb{R} / \mathbb{Z})$ is invertible;

(ii) $\operatorname{det}\left(\Psi_{A}(1)-\right.$ Id $) \neq 0$.

Proof Let $\xi \in \operatorname{ker} \mathcal{L}_{A}$, then $\xi(t)=\Psi(t) \xi_{0}, \xi_{0} \in \mathbb{R}^{2}$. By periodicity $\xi(1)=\Psi(1) \xi_{0}=\xi_{0}$, which implies that $1 \in \sigma(\Psi(1)-$ Id). The implication (i) $\Longrightarrow$ (ii) follows immediately. On the other hand if (ii) holds, then $\operatorname{ker} \mathcal{L}_{A}=\{0\}$. Since $\mathcal{L}_{A}$ is Fredholm with index $\operatorname{ind}\left(\mathcal{L}_{A}\right)=0$ we conclude that coker $\mathcal{L}_{A}=\{0\}$, which establishes the invertibility and completes the implication (ii) $\Longrightarrow$ (i).

Consider a generic path $\eta \mapsto \mathcal{F}_{\Theta}(\eta)$ as described in Definition 1 . Consider the oneparameter family of differential equations $\mathcal{F}_{\Theta}(\eta) \Psi=\frac{d \Psi}{d t}-\mathcal{R}(t ; \eta) \Psi=0$ with $\Psi(0 ; \eta)=$ Id, for all $\eta \in I$, which defines a one-parameter family of loops $t \mapsto \Psi(t ; \eta), \eta \in I$. By definition, $\Psi(t ; 1)=\Psi_{A}(t)$. By construction the path $\mathcal{F}_{\Theta}(\eta)$ intersects $\Sigma_{1}$ transversally at finitely many points $\eta_{0}$.

Lemma 4 The function $\eta \mapsto \operatorname{det}(\Psi(1 ; \eta)-\mathrm{Id})$ changes sign at every intersection point $\eta_{0}$.

Proof At an intersection point $\eta_{0}$, $\operatorname{ker} \mathcal{F}_{\Theta}\left(\eta_{0}\right)$ is one-dimensional and the eigenvectors are spanned by $\xi(t)=\Psi\left(t ; \eta_{0}\right) \xi_{0}$. Consequently, $\operatorname{ker}\left(\Psi\left(1 ; \eta_{0}\right)-\mathrm{Id}\right)$ is one dimensional and thus $\eta \mapsto \operatorname{det}\left(\Psi(1 ; \eta)-\right.$ Id) changes sign at every $\eta_{0}$.

Theorem 3 Let $t \mapsto A(t)$ be a non-degenerate loop of $2 \times 2$ matrices. Then,

$$
\iota(A)=-\operatorname{sgn}\left(\operatorname{det}\left(\Psi_{A}(1)-\mathrm{Id}\right)\right) .
$$

Proof By Lemma 3 the function $\eta \mapsto \operatorname{det}(\Psi(1 ; \eta)-\mathrm{Id})$ has a fixed sign at every $\eta \in I$ for which $\mathcal{F}_{\Theta}(\eta)$ is invertible. By Lemma 4 the intersections points $\eta_{0}$ of $\mathcal{F}_{\Theta}\left(\eta_{0}\right)$ with $\Sigma_{1}$ correspond to sign changes of the function $\eta \mapsto \operatorname{det}(\Psi(1 ; \eta)-$ Id). Therefore, the number of sign changes of the function $\eta \mapsto \operatorname{det}(\Psi(1 ; \eta)-\mathrm{Id})$ is equal to $\gamma$.

Since the definition of $\mathrm{l}(A)$ does not depend on the matrix $\Theta$ we choose $\Theta=$ Id. For $\eta=0$ we have $\Psi(t ; 0)=e^{t} \operatorname{Id}$ and therefore $\operatorname{sgn}(\operatorname{det}(\Psi(1 ; 0)-\mathrm{Id}))=+1$. Consequently, the $\gamma$ consecutive sign changes of $\operatorname{det}(\Psi(1 ; \eta)-\mathrm{Id})$ yield $\operatorname{sgn}(\operatorname{det}(\Psi(1 ; 1)-\mathrm{Id}))=$ $\operatorname{sgn}\left(\operatorname{det}\left(\Psi_{A}(1)-\mathrm{Id}\right)\right)=(-1)^{\gamma}$. Moreover, because $\operatorname{sgn}(\operatorname{det}(\mathrm{Id}))=+1$, we obtain the desired result.

For non-degenerate loops $t \mapsto J S(t)$, with $S(t)$ symmetric matrices for all $t$, the index $\mathrm{\imath}(J S)$ is related to the well-known Conley-Zehnder index of symplectic loops. Consider the differential equation $\frac{d \Psi_{J S}}{d t}-J S(t) \Psi_{J S}=0$, with $\Psi_{J S}(0)=\mathrm{Id}$, which defines the loop $t \mapsto \Psi_{J S}(t)$ of symplectic matrices. By Lemma 3(ii) $\Psi_{J S}(1)$ has no eigenvalues equal to 1 and therefore the Conley-Zehnder index of the symplectic path $\Psi_{J S}(t)$ is well-defined. From [13] we consider the crossing form $\Gamma\left(\Psi_{J S}, t\right)$ defined for vectors $\xi \in \operatorname{ker}\left(\Psi_{J S}(t)-\right.$ Id):

$$
\Gamma\left(\Psi_{J S}, t\right) \xi=\omega\left(\xi, \frac{d}{d t} \Psi_{J S}(t) \xi\right)=(\xi, S(t) \xi) .
$$

A crossing $t_{0}>0$ is defined by $\operatorname{det}\left(\Psi_{J S}\left(t_{0}\right)-\mathrm{Id}\right)=0$ and a crossing is regular if the crossing form is non-singular. A path $t \mapsto \Psi_{J S}(t)$ is regular if all crossings are regular. Any path can be approximated by a regular path with the same endpoints and which is homotopic to the initial path, see [12] for details. For a regular path $t \mapsto \Psi_{J S}(t)$ the Conley-Zehnder index is given by

$$
\mu^{C Z}\left(\Psi_{J S}\right)=\frac{1}{2} \operatorname{sgn} S(0)+\sum_{\substack{t_{0}>0, \operatorname{det}\left(\Psi_{J S}\left(t_{0}\right)-\mathrm{Id}\right)=0}} \operatorname{sgn} \Gamma\left(\Psi_{J S}, t_{0}\right) .
$$


Proposition 1 Let $t \mapsto S(t)$ be a non-degenerate loop of symmetric $2 \times 2$ matrices. Then,

$$
\iota(J S)=(-1)^{\mu}{ }^{C Z}\left(\Psi_{J S}\right) .
$$

Proof From [15, Thm. 3.3] and [14, Sect. 2.4] we have the determinant property for the Conley-Zehnder index: $\operatorname{sgn}\left(\operatorname{det}\left(\Psi_{J S}(1)-\mathrm{Id}\right)\right)=(-1)^{-1+\mu^{C Z}\left(\Psi_{J S}\right)}$. Therefore,

$$
\iota(J S)=-\operatorname{sgn}\left(\operatorname{det}\left(\Psi_{J S}(1)-\mathrm{Id}\right)\right)=-(-1)^{-1+\mu^{C Z}\left(\Psi_{J S}\right)}=(-1)^{\mu^{C Z}\left(\Psi_{J S}\right)},
$$

which completes the proof.

\section{Closed integral curves}

The 1-periodic closed integral curves of a vector field $X \in \mathscr{X}\left(\mathbb{D}^{2} \times \mathbb{R} / \mathbb{Z}\right)$ satisfy the differential equation

$$
\left\{\begin{array}{l}
\frac{d x}{d t}=X(x, t), \quad x \in \mathbb{D}^{2}, t \in \mathbb{R} / \mathbb{Z}, \\
x(0)=x(1) .
\end{array}\right.
$$

Proposition 2 Let $y$ be a skeleton of closed integral curves for $X$ and let $[x$ rel $y]$ be a proper relative braid class. Then, there exists an $0<r<1$ such that

$$
\left|x_{0}(t)\right|<r, \text { and }\left|x_{0}(t)-y^{j}(t)\right|>1-r, \quad \forall j=1, \ldots, m, \quad \forall t \in \mathbb{R},
$$

for all 1-periodic closed integral curves $x_{0}$ such that $x_{0}$ rel $y \in[x]$ rel $y$.

Proof The 1-periodic closed characteristics satisfy the uniform bound $|x(t)| \leq 1$ for all $t \in \mathbb{R} / \mathbb{Z}$. Therefore, $\left|x_{t}(t)\right| \leq c$ for all $t \in \mathbb{R} / \mathbb{Z}$, which yields the a priori bound $\|x\|_{H^{1}} \leq c$. By differentiating the equation we obtain the a priori bound $\|x\|_{H^{2}} \leq c$. By the compact embedding $H^{2}(\mathbb{R} / \mathbb{Z}) \hookrightarrow H^{1}(\mathbb{R} / \mathbb{Z})$ sequences of closed integral curves $\left\{x^{n}\right\}$ have convergent subsequences in $H^{1}(\mathbb{R} / \mathbb{Z})$ and therefore the set of 1-periodic closed integral curves $x_{0}$, such that $x_{0}$ rel $y \in[x]$ rel $y$, is a compact subset in $H^{1}(\mathbb{R} / \mathbb{Z})$.

Assume that an $0<r<1$ as stated in the proposition does not exist. Then, by the above compactness there exists a sequence $x^{n} \rightarrow x_{0}$ such that one, or both of the following two possibilities hold: (i) $\left|x_{0}\left(t_{0}\right)\right|=1$ for some $t_{0}$. By the uniqueness of the initial value problem of Equation (17) and the invariance of the boundary $\partial \mathbb{D}^{2}$ ( $X$ is tangent to the boundary), $\left|x_{0}(t)\right|=1$ for all $t \in \mathbb{R} / \mathbb{Z}$, which is impossible since $[x]$ rel $y$ is proper; (ii) $x_{0}\left(t_{0}\right)=y^{j}\left(t_{0}\right)$ for some $t_{0}$ and some $j$. As before, by the uniqueness of the initial value problem of Equation (17), then $x_{0}(t)=y^{j}(t)$ for all $t \in \mathbb{R} / \mathbb{Z}$, which again contradicts the fact that $[x]$ rel $y$ is proper.

Let $\mathcal{M} \in \operatorname{GL}\left(L^{2}, H^{1}\right)$ be an isomorphism such that $\mathcal{M} \mathscr{E}(x)$ is of the form $\mathcal{M} \mathscr{E}(x)=$ $\Phi_{\mathcal{M}}(x)=x-K_{\mathcal{M}}(x)$, with $K_{\mathcal{M}}: H^{1}(\mathbb{R} / \mathbb{Z}) \rightarrow H^{1}(\mathbb{R} / \mathbb{Z})$ compact. Such an isomorphism $\mathcal{M}$ is called a (constant) parametrix for $\mathscr{E}$. The mapping $\Phi_{\mathcal{M}}: H^{1}(\mathbb{R} / \mathbb{Z}) \rightarrow H^{1}(\mathbb{R} / \mathbb{Z})$ is a Fredholm mapping of index 0 .

Proposition 3 Let $\mathcal{M}$ be a parametrix for $\mathscr{E}$. A function $x_{0} \in H^{1}(\mathbb{R} / \mathbb{Z})$ is a solution of $\Phi_{\mathcal{M}}\left(x_{0}\right)=0$ if and only if $x_{0}$ satisfies Eq. (17).

Proof If $x_{0} \in H^{1}(\mathbb{R} / \mathbb{Z})$ is a solution of Eq. (17), then $\Phi_{\mathcal{M}}\left(x_{0}\right)=0$ is obviously satisfied. On the other hand, if $x_{0} \in H^{1}(\mathbb{R} / \mathbb{Z})$ is a zero of $\Phi_{\mathcal{M}}$, then applying $\mathcal{M}$ to both sides shows that $x_{0}$ satisfies Eq. (17). 
An explicit parametrix can be constructed by considering the linear operator $\mathcal{N}_{\mu}: H^{1}$ $\left(\mathbb{R} / \mathbb{Z} ; \mathbb{R}^{2}\right) \rightarrow L^{2}\left(\mathbb{R} / \mathbb{Z} \mathbb{R}^{2}\right)$, defined by

$$
\mathcal{N}_{\mu}:=-J \frac{d}{d t}+\mu, \quad \mu \in \mathbb{R} .
$$

The operator $\mathcal{N}_{\mu}$ is invertible for $\mu \neq 2 \pi k, k \in \mathbb{Z}$ and the inverse $\mathcal{N}_{\mu}^{-1}: L^{2}\left(\mathbb{R} / \mathbb{Z} ; \mathbb{R}^{2}\right) \rightarrow$ $H^{1}\left(\mathbb{R} / \mathbb{Z} ; \mathbb{R}^{2}\right) \subset L^{2}\left(\mathbb{R} / \mathbb{Z} ; \mathbb{R}^{2}\right)$ is compact. If we choose the explicit parametrix $\mathcal{M}_{\mu}=$ $-\mathcal{N}_{\mu}^{-1} J$, then Eq. (17) transforms to

$$
\Phi(x):=\mathcal{M}_{\mu} \mathscr{E}(x)=x-\mathcal{N}_{\mu}^{-1}(-J X(x, t)+\mu x)=x-K(x),
$$

where $K(x):=\mathcal{N}_{\mu}^{-1}(-J X(x, t)+\mu x): H^{1}(\mathbb{R} / \mathbb{Z}) \rightarrow H^{1}(\mathbb{R} / \mathbb{Z})$ is compact.

\section{The proof of theorems 1}

In order to apply the Leray-Schauder degree theory to the mapping $\Phi$ we need an appropriate domain of definition $\Omega \subset H^{1}(\mathbb{R} / \mathbb{Z})$. Define the $\Omega$ as the subset of functions $x_{0} \in H^{1}(\mathbb{R} / \mathbb{Z})$ such that $x_{0}$ rel $y \in[x]$ rel $y$. If $[x$ rel $y]$ is proper, then Proposition 2 implies that $\Phi^{-1}(0) \cap$ $\partial \Omega=\varnothing$. Combining the latter with the fact that $\Omega$ is a bounded set yields that the LeraySchauder degree $\operatorname{deg}_{L S}(\Phi, \Omega, 0)$ is well-defined.

\subsection{Hamiltonian vector fields}

Recall from (1) that a Hamiltonian vector field is given by $X_{H}(x, t)=J \nabla H(x, t)$, where $H(x, t)$ is a smooth Hamiltonian satisfying Hypotheses (i)-(ii) in Sect. 1.1. In particular, $X_{H} \in$ $\mathscr{X}\left(\mathbb{D}^{2} \times \mathbb{R} / \mathbb{Z}\right)$. Associated with the vector field $X_{H}$ we write $\mathscr{E}_{H}(x)=x_{t}-X_{H}(x(t), t)$ and

$$
\Phi_{H}(x):=\mathcal{M}_{\mu} \mathscr{E}_{H}(x)=x-\mathcal{N}_{\mu}^{-1}\left(-J X_{H}(x, t)+\mu x\right)=x-\mathcal{N}_{\mu}^{-1}(\nabla H(x, t)+\mu x) .
$$

Let $y$ be a skeleton for $X$. We can construct a Hamiltonian $H$ such that $y$ is also a skeleton for the associated vector field $X_{H}$, cf. [17, Lemma 6.10]. The set of such Hamiltonians is denoted by $\mathscr{H}(y)$. From [17, Prop. 9.1] we can choose $H$ generically such that all zeroes in $\Phi_{H}^{-1}(0) \cap \Omega=\operatorname{Crit}_{\mathscr{A}_{H}}([x] \mathrm{rel} y)$ are non-degenerate. The Leray-Schauder degree is given by

$$
\operatorname{deg}_{L S}\left(\Phi_{H}, \Omega, 0\right)=\sum_{x \in \Phi_{H}^{-1}(0) \cap \Omega} \operatorname{deg}_{L S}\left(\Phi_{H}, B_{\epsilon}(x), 0\right)=\sum_{x \in \Phi_{H}^{-1}(0) \cap \Omega} \operatorname{deg}_{L S}\left(D_{x} \Phi_{H}(x)\right),
$$

where $B_{\epsilon}(x)$ is a sufficiently small $H^{1}$-neighborhood of $x$ and $\operatorname{deg}_{L S}\left(D_{x} \Phi_{H}(x)\right)$ is local degree with

$$
D_{x} \Phi_{H}(x)=\mathcal{M}_{\mu} D_{x} \mathscr{E}_{H}(x)=\mathcal{M}_{\mu}\left(\frac{d}{d t}-J S(t)\right),
$$

and $S(t)=D_{x}^{2} H(x(t), t)$.

Lemma 5 Let $\theta>0$, then $\beta_{\mathcal{M}_{\mu}}(\theta J)=2\left\lceil\frac{\mu+\theta}{2 \pi}\right\rceil$.

Proof The eigenvalues of the operator Id $-(\theta+\mu) \mathcal{N}_{\mu}^{-1}$ are given by $\lambda=\frac{-\theta+2 k \pi}{\mu+2 k \pi}$ and all have multiplicity 2 . Therefore number of integers $k$ for which $\lambda<0$ is equal to $\left\lceil\frac{\mu+\theta}{2 \pi}\right\rceil$ and consequently $\beta_{\mathcal{M}_{\mu}}(\theta J)=2\left\lceil\frac{\mu+\theta}{2 \pi}\right\rceil$. 
Let $x_{0} \in \operatorname{Crit}_{\mathscr{A}_{H}}([x]$ rel $y)$. With the choice $\Theta=\theta J$, Lemma 1 and Proposition 1 imply that

$$
\begin{aligned}
\iota\left(\Psi_{J S}\right) & =(-1)^{\mu_{C Z}\left(\Psi_{J S}\right)}=-\operatorname{sgn}(\operatorname{det}(\theta J)) \cdot(-1)^{\beta_{\mathcal{M}_{\mu}}(\theta J)} \operatorname{deg}_{L S}\left(D_{x} \Phi_{H}\left(x_{0}\right)\right) \\
& =-\operatorname{deg}_{L S}\left(D_{x} \Phi_{H}\left(x_{0}\right)\right) .
\end{aligned}
$$

Combining the latter with the Leray-Schauder degree gives

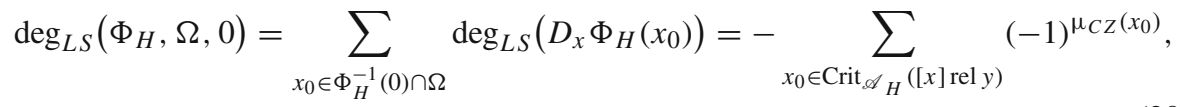

where

$$
\mu_{C Z}\left(x_{0}\right):=\mu_{C Z}\left(\Psi_{J S}\right)
$$

is the Conley-Zehnder index $x_{0}$. By substituting $\tau=-1$ in Eq. (4) and using the expression for the Euler-Floer characteristic in (8) we obtain

$$
\chi(x \text { rel } y)=\sum_{x_{0} \in \operatorname{Crit} \mathscr{A}_{H}([x] \text { rel } y)}(-1)^{\mu^{C Z}\left(x_{0}\right)} .
$$

If we combine the degree formula in (20) and Eq. (22) we obtain:

$$
\operatorname{deg}_{L S}\left(\Phi_{H}, \Omega, 0\right)=-\chi(x \operatorname{rel} y) .
$$

\subsection{The Poincaré-Hopf index formula}

Lemma 6 For an arbitrary choice of $\mathcal{M}$ and $\Theta$ the index $\mathrm{L}(x)$ of an isolated zero of $\mathscr{E}$ is given by

$$
\iota(x):=-\operatorname{sgn}(\operatorname{det}(\Theta))(-1)^{\beta_{\mathcal{M}}(\Theta)} \operatorname{deg}_{L S}\left(\Phi_{\mathcal{M}}, B_{\epsilon}(x), 0\right),
$$

where $\epsilon>0$ is chosen sufficiently small such that $x$ is the only zero of $\mathscr{E}$ in $B_{\epsilon}(x) \subset H^{1}(\mathbb{R} / \mathbb{Z})$. The expression for $\mathrm{I}(x)$ is independent of choices of $\mathcal{M}$ and $\Theta$.

Proof By the Sard-Smale Theorem one can choose an arbitrarily small $h \in L^{2}(\mathbb{R} / \mathbb{Z})$, $\|h\|_{L^{2}}<\delta$, such that $h$ is a regular value of $\mathscr{E}$ and $\mathscr{E}^{-1}(h) \cap B_{\epsilon}(x)$ consists of finitely many non-degenerate zeroes $x_{h}$. Set $\widetilde{E}(x)=\mathscr{E}(x)-h$ and define

$$
\iota(x):=\sum_{x_{h} \in \tilde{E}^{-1}(0) \cap B_{\epsilon}(x)} \imath\left(x_{h}\right) .
$$

To show that $\mathrm{l}(x)$ is well-defined we choose a parametrix $\mathcal{M}$ (for $\mathscr{E}$ ) and a matrix $\Theta \in$ $\mathrm{M}_{2 \times 2}(\mathbb{R})$ with $\sigma(\Theta) \cap 2 \pi i \mathbb{Z}=\varnothing$ and define $\widetilde{\Phi}_{\mathcal{M}}=\mathcal{M} \widetilde{\mathscr{E}}$. By Lemmas 1 and $2, \mathrm{l}\left(x_{h}\right)=$ $-\operatorname{sgn}(\operatorname{det}(\Theta))(-1)^{\beta_{\mathcal{M}}(\Theta)} \sum_{x_{h}} \operatorname{deg}_{L S}\left(D_{x} \widetilde{\Phi}_{\mathcal{M}}\left(x_{h}\right)\right)$ is independent of $\mathcal{M}$ and $\Theta$. Then,

$$
\begin{aligned}
\sum_{x_{h}} \mathrm{l}\left(x_{h}\right) & =-\operatorname{sgn}(\operatorname{det}(\Theta))(-1)^{\beta_{\mathcal{M}}(\Theta)} \sum_{x_{h}} \operatorname{deg}_{L S}\left(D_{x} \widetilde{\Phi}_{\mathcal{M}}\left(x_{h}\right)\right) \\
& =-\operatorname{sgn}(\operatorname{det}(\Theta))(-1)^{\beta_{\mathcal{M}}(\Theta)} \sum_{x_{h}} \operatorname{deg}_{L S}\left(\widetilde{\Phi}_{\mathcal{M}}, B_{\epsilon_{h}}\left(x_{h}\right), 0\right),
\end{aligned}
$$

where $B_{\epsilon_{h}}\left(x_{h}\right) \subset B_{\epsilon}(x)$ are sufficiently small neighborhoods containing one zero $x_{h}$ only. From the properties of the Leray-Schauder degree we derive that

$$
\sum_{x_{h}} \operatorname{deg}_{L S}\left(\widetilde{\Phi}_{\mathcal{M}}, B_{\epsilon_{h}}\left(x_{h}\right), 0\right)=\operatorname{deg}_{L S}\left(\widetilde{\Phi}_{\mathcal{M}}, B_{\epsilon}(x), 0\right)=\operatorname{deg}_{L S}\left(\Phi_{\mathcal{M}}, B_{\epsilon}(x), 0\right),
$$


which proves the independence of $h$ and thereby the lemma.

Since $y$ is a skeleton for both $X$ and $X_{H}$, it is also a skeleton for the linear homotopy $X_{\alpha}=(1-\alpha) X+\alpha X_{H}, \alpha \in[0,1]$. Define

$$
\Phi_{\alpha}(x):=x-\mathcal{N}_{\mu}^{-1}\left(-J X_{\alpha}(x, t)+\mu x\right)=x-K_{\alpha}(x), \quad \alpha \in[0,1],
$$

with $K_{\alpha}(x)=\mathcal{N}_{\mu}^{-1}\left(-J X_{\alpha}(x, t)+\mu x\right)$. Proposition 2 applies uniformly for all $\alpha \in[0,1]$, i.e. by compactness there exists a uniform $0<r<1$ such that

$$
|x(t)|<r \text {, and }\left|x(t)-y^{j}(t)\right|>1-r,
$$

for all $t \in \mathbb{R}$, for all $j$, for all $x \in \Phi_{\alpha}^{-1}(0) \cap \Omega=\left\{x \in \Omega \mid x=K_{\alpha}(x)\right\}$ and for all $\alpha \in[0,1]$. This implies that the Leray-Schauder degtee $\operatorname{deg}_{L S}\left(\Phi_{\alpha}, \Omega, 0\right)$ is well-defined for all $\alpha \in[0,1]$, and by Eq. (23) and the homotopy invariance of the Leray-Schauder degree we have

$$
\operatorname{deg}_{L S}(\Phi, \Omega, 0)=\operatorname{deg}_{L S}\left(\Phi_{\alpha}, \Omega, 0\right)=\operatorname{deg}_{L S}\left(\Phi_{H}, \Omega, 0\right)=-\chi(x \text { rel } y),
$$

where $\Phi_{0}=\Phi$ and $\Phi_{1}=\Phi_{H}$. Corollary 1 follows from Eq. (25).

Since the expression for $\mathrm{I}(x)$ in Lemma 6 is independent of $\mathcal{M}$ and $\Theta$ we choose $\mathcal{M}=$ $\mathcal{M}_{\mu}=-\mathcal{N}_{\mu}^{-1} J$ and $\Theta={ }_{\theta} J$ as before. Suppose all zeroes of $\mathscr{E}$ in $\Omega$ are isolated. Then, the sum property of the Leray-Schauder degree, Lemma 6 and Eq. (25) imply that

$$
\sum_{x \in \mathscr{E}^{-1}(0) \cap \Omega} \mathrm{l}(x)=-\sum_{x \in \mathscr{E}^{-1}(0) \cap \Omega} \operatorname{deg}_{L S}\left(\Phi, B_{\epsilon}(x), 0\right)=-\operatorname{deg}_{L S}(\Phi, \Omega, 0)=\chi(x \text { rel } y),
$$

which completes the proof Theorem 1 .

\section{Braids on $\mathbb{R}^{2}$, Legendrian braids and braid diagrams}

In this section we show that every relative braid class may be represented by special representatives of Legendrian type. In the forthcoming sections these representatives are used to relate the Euler-Floer characteristic to discrete braid invariants which makes it possible to compute the Euler-Floer characteristic without computing Braid Floer homology.

The braid classes defined in Sect. 1 take values in $\mathbb{D}^{2}$ and are denoted by $[x$ rel $y]$ and $[x]$ rel $y$ respectively. If we consider closed loops in $\mathbb{R}^{2}$, then the relative braid classes on $\mathbb{R}^{2}$ will be denoted by $[x \text { rel } y]_{\mathbb{R}^{2}}$ and $[x]_{\mathbb{R}^{2}}$ rel $y$. A relative braid class on $\mathbb{R}^{2}$ is proper if components $x_{c} \subset x$ cannot be deformed onto (i) itself, or other components $x_{c}^{\prime} \subset x$, or (ii) components $y_{c} \subset y$. A fiber $[x]_{\mathbb{R}^{2}}$ rel $y$ is not bounded!

A relative braid class $[x$ rel $y]$ is positive if it can be represented by positive braids $x^{+}$rel $y^{+}$, i.e. braids with only positive crossings. Braid Floer homology only carries information about positive braid classes. Indeed, every relative braid $x$ rel $y$ may be composed with a sufficient number of positive full twists such that the resulting braid is positive, $\mathrm{cf}$. [17]. By Property (c) of Braid Floer homology (Sect. 1.1) we have $\operatorname{HB}_{k}\left(\left[(x \operatorname{rel} y) \cdot \Delta^{2 \ell}\right]\right) \cong$ $\mathrm{HB}_{k-2 \ell}([x \mathrm{rel} y])$ for all $k \in \mathbb{Z}$. In particular, the Euler-Floer characteristic remains unchanged after composition with full twists. We denote a positive representative $x^{+}$rel $y^{+}$ again by $x$ rel $y$.

Define an augmented skeleton $y^{*}$ by adding the constant strands $y_{-}(t)=(0,-1)$ and $y_{+}(t)=(0,1)$. Braid Floer homology does not change under augmentation, i.e. for proper braid classes it holds that $\mathrm{HB}_{*}([x$ rel $y])=\mathrm{HB}_{*}\left(\left[x\right.\right.$ rel $\left.\left.y^{*}\right]\right)$. For notational simplicity we 
denote the augmented skeleton again by $y$. We also choose the representative $x$ rel $y$ with the additional the property that the projection $\pi_{2} x$ rel $\pi_{2} y$ onto the $q$-coordinate is a relative braid diagram, i.e. there are no tangencies between the strands in the braid diagram. Representatives with the above properties will be referred to as regular positive braids. We denote the projection onto the $q$-coordinate by $q$ rel $Q$, where $q=\pi_{2} x$ and $Q=\pi_{2} y$. The associated regular braids of the form $x_{L}=\left(q_{t}, q\right)$ and $y_{L}=\left(Q_{t}, Q\right)$ are called Legendrian braids with respect to contact form $\theta=p d t-d q$. The Legendrian braids $x_{L}$ rel $y_{L}$ in $[x \text { rel } y]_{\mathbb{R}^{2}}$ project to an equivalence class of braid diagrams denoted by $[q$ rel $Q]$ and is called the class of relative braid diagrams induced by $[x$ rel $y]$. The associated fibers are denoted by $[q] \operatorname{rel} Q$.

Lemma 7 Every regular positive braid $x$ rel $y$ is isotopic to $x_{L}$ rel $y_{L}$ as braids on $\mathbb{R}^{2}$. Moreover, if $x_{L}$ rel $y_{L}$ and $x_{L}^{\prime}$ rel $y_{L}^{\prime}$ are isotopic Legrendrian braids, then they are isotopic via a Legendrian isotopy.

Proof By assumption $x$ rel $y$ is a representative for which the braid diagram $q$ rel $Q$ has only positive transverse crossings. Due to the transversality of intersections the associated Legendrian braid $x_{L}$ rel $y_{L}$, with $x_{L}=\left(q_{t}, q\right)$ and $y_{L}=\left(Q_{t}, Q\right)$, is a braid in $[x \text { rel } y]_{\mathbb{R}^{2}}$. Consider the homotopy $\zeta^{j}(t, \tau)=\tau p^{j}(t)+(1-\tau) q_{t}^{j}$, for every strand $q^{j}$. At $q$-intersections, i.e. times $t_{0}$ such that $q^{j}\left(t_{0}\right)=q^{j^{\prime}}\left(t_{0}\right)$ for some $j \neq j^{\prime}$, it holds that $p^{j}\left(t_{0}\right)-p^{j^{\prime}}\left(t_{0}\right)$ and

$q_{t}^{j}\left(t_{0}\right)-q_{t}^{j^{\prime}}\left(t_{0}\right)$ are non-zero and have the same sign since all crossings in $x$ rel $y$ are positive! Therefore, $\zeta^{j}\left(t_{0}, \tau\right) \neq \zeta^{j^{\prime}}\left(t_{0}, \tau\right)$ for any intersection $t_{0}$ and any $\tau \in[0,1]$, which shows that $x$ rel $y$ and $x_{L}$ rel $y_{L}$ are isotopic.

\section{Mechanical Hamiltonian systems}

\subsection{Hyperbolic Hamiltonians on $\mathbb{R}^{2}$}

Hamiltonian functions of the form

$$
H(x, t)=\frac{1}{2} p^{2}-\frac{1}{2} q^{2}+h(x, t),
$$

where $h$ satisfies the following hypotheses:

(h1) $h \in C^{\infty}\left(\mathbb{R}^{2} \times \mathbb{R} / \mathbb{Z}\right)$;

(h2) $\operatorname{supp}(h) \subset \mathbb{R} \times[-R, R] \times \mathbb{R} / \mathbb{Z}$, for some $R>0$;

(h3) $h$ is a bounded function on $\mathbb{R}^{2} \times \mathbb{R} / \mathbb{Z}$ with all its derivatives up to order 2 .

are called hyperbolic Hamiltonians.

Lemma 8 Let $H$ be a hyperbolic Hamiltonian. Then, there exists a constant $R^{\prime} \geq R>0$, such any 1-periodic closed integral curve $x$ of $X_{H}(x, t)$ satisfies the estimate

$$
|x(t)| \leq R^{\prime}, \text { for all } t \in \mathbb{R} / \mathbb{Z} .
$$

Proof The Hamilton equations in local coordinates are given by

$$
p_{t}=q-h_{q}(p, q, t), \quad q_{t}=p+h_{p}(p, q, t) .
$$

By Hypothesis (h1) we can rewrite the equations as

$$
q_{t t}=h_{p q}(p, q, t) q_{t}+\left(1+h_{p p}(p, q, t)\right)\left(q-h_{q}(p, q, t)\right)+h_{p t}(p, q, t) .
$$


Let $x(t)$ be a closed integral curve of period 1. Suppose there exists a non-trivial interval $I=\left[t_{0}, t_{1}\right] \subset[0,1]$ such that $|q(t)| \geq R$ on $I$ and $\left.|q(t)|\right|_{\partial I}=R$. By Hypothesis (h2) the function $\left.q\right|_{I}$ satisfies the equation $q_{t t}-q=0$. If $\left.q\right|_{I} \geq R$, then $q_{t}\left(t_{0}\right) \geq 0$ and $q_{t}\left(t_{1}\right) \leq 0$. This implies that $0 \geq\left. q_{t}\right|_{\partial I}=\int_{I} q \geq R|I|>0$, which is a contradiction. The same argument holds for $\left.q\right|_{I} \leq-R$. We conclude that

$$
|q(t)| \leq R, \quad \text { for all } t \in \mathbb{R} / \mathbb{Z} .
$$

We now use the a priori $q$-estimate in combination with Eq. (27) and Hypothesis (h3). Multiplying Eq. (27) by $-q$ and integrating over [0,1] gives:

$$
\begin{aligned}
\int_{0}^{1} q_{t}^{2} & =-\int_{0}^{1} h_{p q} q_{t} q-\int_{0}^{1}\left(1+h_{p p}\right)\left(q-h_{q}\right) q-\int_{0}^{1} h_{p t} q \\
& \leq C \int_{0}^{1}\left|q_{t}\right|+C \leq \epsilon \int_{0}^{1} q_{t}^{2}+C_{\epsilon},
\end{aligned}
$$

which implies that $\int_{0}^{1} q_{t}^{2} \leq C(R)$. The $L^{2}$-norm of the right hand side in (27) can be estimated using the $L^{\infty}$-estimate on $q$ and the $L^{2}$-estimate on $q_{t}$, which yields $\int_{0}^{1} q_{t t}^{2} \leq C(R)$. Combining these estimates we have that $\|q\|_{H^{2}(\mathbb{R} / \mathbb{Z})} \leq C(R)$ and therefore $\left|q_{t}(t)\right| \leq C(R)$ for all $t \in \mathbb{R} / \mathbb{Z}$. From the Hamilton equations it follows that $|p(t)| \leq\left|q_{t}(t)\right|+C$, which proves the lemma.

A (smooth) homotopy of Hamiltonians $H(x, t ; \alpha)$, satisfying (h1)-(h3) with uniform constants $R>0$ and $c>0$, for all $\alpha \in[0,1]$, is called a homotopy of hyperbolic Hamiltonians. Closed integral curves of the Hamiltonian vector fields given by $H(x, t ; \alpha)$ are denoted by $x_{\alpha}$.

Lemma 9 Let $H(x, t ; \alpha), \alpha \in[0,1]$ be (smooth) homotopy of hyperbolic Hamiltonians. Then, there exists a uniform constant $R^{\prime} \geq R$ such that any 1-periodic closed integral curve $x_{\alpha}$ satisfies $\left|x_{\alpha}(t)\right| \leq R^{\prime}$ for all $t \in \mathbb{R} / \mathbb{Z}$ and for all $\alpha \in[0,1]$.

Proof The a priori $H^{2}$-estimates in Lemma 8 holds with uniform constants with respect to $\alpha \in[0,1]$. The the remainder of the proof remains unchanged.

\subsection{Hamiltonians of mechanical type}

Consider hyperbolic Hamiltonians of the form $H_{L}(x, t)=\frac{1}{2} p^{2}-\frac{1}{2} q^{2}+g(q, t)$, which are referred to as hyperbolic Hamiltonians of mechanical type. On the potential functions $g$ we impose the following hypotheses:

(g1) $g \in C^{\infty}(\mathbb{R} \times \mathbb{R} / \mathbb{Z})$;

(g2) $\operatorname{supp}(g) \subset[-R, R] \times \mathbb{R} / \mathbb{Z}$, for some $R>1$.

A Legendrian braid $y_{L}$ has linear crossings if the following property holds. Let $t=t_{0}$ be a crossing and let $I\left(t_{0}\right)$ be the set of labels defined by: $i, j \in I\left(t_{0}\right)$, if $i \neq j$ and $Q^{i}\left(t_{0}\right)=Q^{j}\left(t_{0}\right)$. A crossing at $t=t_{0}$ is linear if

$$
Q_{t}^{i}(t)=\text { constant }, \quad \forall i \in I\left(t_{0}\right), \text { and } \forall t \in\left(-\epsilon+t_{0}, \epsilon+t_{0}\right),
$$

for some $\epsilon=\epsilon\left(t_{0}\right)>0$.

Every skeleton $Q$ with transverse crossings is isotopic to a skeleton with linear crossings via a small local deformation at crossings. Therefore, every regular positive braid $y$ is isotopic to a Legendrian braid $y_{L}$ with linear crossings. For Legendrian braids $x_{L}$ rel $y_{L} \in[x \text { rel } y]_{\mathbb{R}^{2}}$ with linear crossings the following result holds: 
Lemma 10 Let $y_{L}$ be a Legendrian skeleton with linear crossings. Then, there exists a hyperbolic Hamiltonian of the form $H_{L}(x, t)=\frac{1}{2} p^{2}-\frac{1}{2} q^{2}+g(q, t)$, with $g$ satisfying Hypotheses $(g 1)-(g 2)$, and $R>0$ sufficiently large, such that $y_{L}$ is a skeleton for $X_{H_{L}}(x, t)$.

Proof The linear crossings in $y_{L}$ allow us to follow the construction in [17]. For each strand $Q^{i}$ we define the potentials $g^{i}(t, q)=-Q_{t t}^{i}(t) q$. By construction $Q^{i}$ is a solution of the equation $Q_{t t}^{i}=-g_{q}^{i}\left(t, Q^{i}\right)$. Now choose small tubular neighborhoods of the strands $Q^{i}$ and cut-off functions $\omega^{i}$ that are equal to 1 near $Q^{i}$ and are supported in the tubular neighborhoods. If the tubular neighborhoods are narrow enough, then $\operatorname{supp}\left(\omega^{i} g^{i}\right) \cap \operatorname{supp}\left(\omega^{j} g^{j}\right)=\varnothing$, for all $i \neq j$, due to the fact that at crossings the functions $g^{i}$ in question are zero. This implies that all strands $Q^{i}$ satisfy the differential equation $Q_{t t}^{i}=-\sum_{i} \omega^{j}(t) g_{q}^{j}\left(Q^{i}, t\right)$. On $[-1,1] \times \mathbb{R} / \mathbb{Z}$, the function is $\sum_{i} \omega^{i}(t) g^{i}(q, t)$ is compactly supported. The latter follows from the fact that for the constant strands $Q^{i}= \pm 1$, the potentials $g^{i}$ vanish. Let $R>1$ and define

$$
\tilde{g}^{i}(t, q)= \begin{cases}g^{i}(t, q) & \text { for }|q| \leq 1, t \in \mathbb{R} / \mathbb{Z}, \\ -\frac{1}{2 m} q^{2} & \text { for }|q| \geq R, t \in \mathbb{R} / \mathbb{Z} .\end{cases}
$$

where $m=\# Q$, which yields smooth functions $\tilde{g}^{i}$ on $\mathbb{R} \times \mathbb{R} / \mathbb{Z}$. Define

$$
g(q, t)=\frac{1}{2} q^{2}+\sum_{i=1}^{m} \tilde{g}^{i}(q, t) .
$$

By construction $\operatorname{supp}(g) \subset[-R, R] \times \mathbb{R} / \mathbb{Z}$, for some $R>1$ and the strands $Q^{i}$ all satisfy the Euler-Lagrange equations $Q_{t}^{i}=Q^{i}-g_{q}\left(Q^{i}, t\right)$, which completes the proof.

\section{The Poincaré-Hopf index formula for Lagrangian systems}

Lagrangian systems with Lagrangians of the form $L(q, t)=\frac{1}{2} q_{t}^{2}-V(q, t)$ are equivalent to Hamiltonian systems with mechanical Hamiltonians of the form $H(x, t)=\frac{1}{2} p^{2}+V(q, t)$. There is a relation between the Morse index for Lagrangians systems and the Conley-Zehnder index for Hamiltonians systems in this setting.

\subsection{The Lagrangian Morse index}

Consider the Lagrangian action $\mathscr{L}(q)=\int_{0}^{1} \frac{1}{2} q_{t}^{2}-V(q, t) d t$. The linearization at a critical points $q(t)$ of the Lagrangian action is given by the unbounded operator

$$
\mathcal{C}=-\frac{d^{2}}{d t^{2}}-D_{q}^{2} V(q(t), t): H^{2}(\mathbb{R} / \mathbb{Z}) \subset L^{2}(\mathbb{R} / \mathbb{Z}) \rightarrow L^{2}(\mathbb{R} / \mathbb{Z}) .
$$

A critical point $q(t)$ is non-degenerate if the above operator is invertible. Define the 'Lagrangian' Morse index $\gamma_{\mathcal{e}}$ as the number of negative eigenvalues of $\mathcal{C}$. ${ }^{4}$ The Morse index of a critical point $q$ of $\mathscr{L}$ is defined as $\gamma(q):=\gamma_{\mathrm{e}}$.

Consider a path of unbounded self-adjoint operators on $L^{2}(\mathbb{R} / \mathbb{Z})$ given by $\eta \mapsto \mathcal{C}(\eta)=$ $-\frac{d^{2}}{d t^{2}}-\mathcal{Q}(t ; \eta)$, with $\mathcal{Q}(t ; \eta)$ smooth. The path $\eta \mapsto \mathcal{C}(\eta)$ is continuously differentiable in the (weak) operator topology of $\mathscr{B}\left(H^{2}, L^{2}\right)$ and Hypotheses (A1)-(A3) in [13] are satisfied. We now repeat the definition of spectral flow for a path of unbounded operators as developed

4 We use the adjective 'Lagrangian' to distinguish the latter from the Morse index in (12). 
in [13]. The crossing operator for a path $\eta \mapsto \mathcal{C}(\eta)$ is given by $\Gamma(\mathcal{C}, \eta)=\left.\pi \frac{d}{d \eta} \mathcal{C}(\eta) \pi\right|_{\text {ker }} \mathcal{C}(\eta)$, where $\pi$ is the orthogonal projection onto $\operatorname{ker} C(\eta)$. A crossing $\eta_{0} \in I$ is a number for which the operator $C\left(\eta_{0}\right)$ is not invertible. A crossing is regular if $\Gamma\left(\mathcal{B}, \eta_{0}\right)$ is non-singular. A point $\eta_{0}$ for which $\operatorname{dim} \operatorname{ker} \mathcal{C}\left(\eta_{0}\right)=1$, is called a simple crossing. A path $\eta \mapsto \mathcal{C}(\eta)$ is called generic if all crossing are simple and every path $\eta \mapsto \mathcal{C}(\eta)$ with invertible ends is homotopic to a generic path. At a simple crossing $\eta_{0}$ there exists a $C^{1}$-curve $\ell(\eta)$, for $\eta$ near $\eta_{0}$, and $\ell(\eta)$ is an eigenvalue of $\varrho(\eta)$ with $\ell\left(\eta_{0}\right)=0$ and $\ell^{\prime}\left(\eta_{0}\right) \neq 0$. The spectral flow for a generic path is defined by

$$
\operatorname{specflow}(\mathcal{C}(\eta), I)=\sum_{\ell\left(\eta_{0}\right)=0} \operatorname{sgn}\left(\ell^{\prime}\left(\eta_{0}\right)\right),
$$

and at simple crossings $\eta_{0}$,

$$
\Gamma(\mathcal{C}, \eta) \phi\left(\eta_{0}\right)=\left(\frac{d}{d \eta} \mathcal{C}\left(\eta_{0}\right) \phi\left(\eta_{0}\right), \phi\left(\eta_{0}\right)\right)_{L^{2}} \phi\left(\eta_{0}\right)=\ell^{\prime}\left(\eta_{0}\right) \phi\left(\eta_{0}\right),
$$

after normalizing $\phi\left(\eta_{0}\right)$ in $L^{2}(\mathbb{R} / \mathbb{Z})$. The derivative of $\ell$ at $\eta_{0}$ is given by

$$
\ell^{\prime}\left(\eta_{0}\right)=-\left(\partial_{\eta} \mathcal{S}\left(t ; \eta_{0}\right) \phi\left(\eta_{0}\right), \phi\left(\eta_{0}\right)\right)_{L^{2}} .
$$

Proposition 4 Assume that the endpoints of $\eta \mapsto \mathcal{C}(\eta)$ are invertible. Then,

$$
\operatorname{specflow}(\mathcal{C}(\eta), I)=\gamma_{\mathcal{C}(0)}-\gamma_{\mathcal{C}(1)}
$$

Proof Let $c>0$ be a sufficiently large constant such that $\mathcal{C}(0)+c$ Id and $\mathcal{C}(1)+c$ Id are positive definite self-adjoint operators on $L^{2}(\mathbb{R} / \mathbb{Z})$. Consider the paths $\eta \mapsto \mathcal{C}_{1}(\eta)=$ $\mathcal{C}(0)+c \eta \operatorname{Id}$ and $\eta \mapsto \mathcal{C}_{2}(\eta)=\mathcal{C}(1)+c(1-\eta) \mathrm{Id}$. Their concatenation $\mathcal{C}_{1} \# \mathcal{C}_{2}$ is a path from $\mathcal{C}(0)$ to $\mathcal{C}(1)$ and $\eta \mapsto \mathcal{C}_{1} \# \mathcal{C}_{2}$ is homotopic to $\eta \mapsto \mathcal{C}(\eta)$. Using the homotopy invariance and the concatenation property of the spectral flow (cf. [13]) we obtain

$\left.\operatorname{specflow}\left(C_{(\eta)}\right), I\right)=\operatorname{specflow}\left(\complement_{1} \# \bigodot_{2}, I\right)=\operatorname{specflow}\left(\bigodot_{1}, I\right)+\operatorname{specflow}\left(\bigodot_{2}, I\right)$.

Since $\mathcal{C}(0)$ is invertible, the regular crossings of $\mathcal{C}_{1}(\eta)$ are given by $\eta_{i}^{1}=-\frac{\lambda_{i}}{c}$, where $\lambda_{i}$ are negative eigenvalues of $\mathcal{C}(0)$. By the positive definiteness of $\mathcal{C}(0)+c \mathrm{Id}$, the negative eigenvalues of $\mathcal{C}(0)$ satisfy $0>\lambda_{i}>-c$. For the crossings $\eta_{i}$ this implies

$$
0<\eta_{i}=-\frac{\lambda_{i}}{c}<1,
$$

and therefore the number of crossings equals the number of negative eigenvalues of $\mathcal{C}(0)$ counted with multiplicity. By the choice of $c$, we also have that $\frac{d}{d \eta} \varrho_{1}(\eta)=c$ Id is positive definite and therefore the signature of the crossing operator of $\mathcal{C}_{1}(\eta)$ is exactly the number of negative eigenvalues of $\mathcal{C}(0)$, i.e. $\operatorname{specflow}\left(\mathcal{C}_{1}, I\right)=\gamma_{\mathcal{C}(0)}$. For $\mathcal{C}_{2}(\eta)$ we obtain, $\operatorname{specflow}\left(\mathcal{C}_{2}, I\right)=-\gamma_{\mathcal{C}(1)}$. This proves that specflow $(\mathcal{C}(\eta), I)=\gamma_{\mathcal{C}(0)}-\gamma_{\mathcal{C}(1)}$.

Lemma 11 (cf. [1] and [2]) Let q be a non-degenerate critical point of $\mathscr{L}$. Then, the ConleyZehnder index of the associated critical point $x=\left(q_{t}, q\right)$ of the Hamiltonian action $\mathscr{A}_{H}$ is well-defined and $\mu^{C Z}(x)=\gamma(q)$.

Proof Consider the curves $\eta \mapsto \mathcal{B}(\eta)$ and $\eta \mapsto \mathcal{C}(\eta), \eta \in I=[0,1]$ given by

$$
\mathcal{B}(\eta)=-J \frac{d}{d t}-\left(\begin{array}{cc}
1 & 0 \\
0 & \mathcal{Q}(t ; \eta)
\end{array}\right), \quad \mathcal{C}(\eta)=-\frac{d^{2}}{d t^{2}}-\mathcal{Q}(t ; \eta)
$$

The crossing forms of the curves are the same $-\Gamma(\mathcal{B}, \eta)=\Gamma(\mathcal{C}, \eta)$ - and therefore also the crossings $\eta_{0}$ are identical. Indeed, $\mathcal{B}\left(\eta_{0}\right)$ is non-invertible if and only if $\mathcal{C}\left(\eta_{0}\right)$ is non-invertible 
and consequently specflow $(\mathcal{B}(\eta), I)=\operatorname{specflow}(\mathcal{C}(\eta), I)$. By [12], $\operatorname{specflow}(\mathcal{B}(\eta), I)=$ $\mu_{\mathcal{B}(0)}^{C Z}-\mu_{\mathcal{B}(1)}^{C Z}$ and by Proposition 4, specflow $(\mathcal{C}(\eta), I)=\gamma_{\mathcal{C}(0)}-\gamma_{\mathcal{C}(1)}$, which implies that

$$
\gamma_{\mathcal{C}(0)}-\gamma_{\mathcal{C}(1)}=\mu_{\mathcal{B}(0)}^{C Z}-\mu_{\mathcal{B}(1)}^{C Z}
$$

where $\mu_{\mathcal{B}(0)}^{C Z}$ and $\mu_{\mathcal{B}(1)}^{C Z}$ are the Conley-Zehnder indices of the symplectic paths defined by $\mathcal{B}(0)$ and $\mathcal{B}(1)$ respectively, cf. (15). Now choose $\mathcal{Q}(t ; \eta)$ such that $\mathcal{Q}(t ; 0)=D_{q}^{2} V(q(t), t)+$ $c$ and $\mathcal{Q}(t ; 1)=D_{q}^{2} V(q(t), t)$ and such that $\eta \mapsto \mathcal{B}(\eta)$ and $\eta \mapsto \mathcal{C}(\eta)$ are regular curves. If $c \ll 0$, then $\gamma_{\mathcal{C}(0)}=0$. In order to compute $\mu_{\mathcal{B}(0)}^{C Z}$ we invoke the crossing from $\Gamma(\Psi, t)$ for the associated symplectic path $\Psi(t)$, cf. Eq. (14). Crossings at $t_{0} \in(0,1]$ correspond to non-trivial solutions of the equation $\mathrm{C}(0) \psi=0$ on $\left[0, t_{0}\right]$, with periodic boundary conditions. To be more precise, let $\Psi=(\phi, \psi)$, then $\mathcal{B}(0) \Psi=0$ is equivalent to $\psi_{t}=\phi$ and $-\phi_{t}-$ $\left(D_{q}^{2} V(q(t), t)+c\right) \psi=0$, which yields the equation $\mathcal{C}(0) \psi=0$. For the latter the kernel is trivial for any $t_{0} \in(0,1]$. Indeed, if $\psi$ is a solution, then $\int_{0}^{t_{0}}\left|\psi_{t}\right|^{2}=\int_{0}^{t_{0}}\left(D_{q}^{2} V(q, t)+c\right) \psi^{2}<$ 0 , which is a contradiction. Therefore, there are no crossing $t_{0} \in(0,1]$. As for $t_{0}=0$ we have that $D_{q}^{2} V(q(0), 0)+c$ Id $<0$, which implies that $\operatorname{sgn} \mathcal{S}(0 ; 0)=0$ and therefore $\mu_{\mathcal{B}(0)}^{C Z}=0$, which proves the lemma.

\subsection{The Poincaré-Hopf index formula}

The Hamiltonian $H_{L}$ given by Lemma 10 gives rise to a Lagrangian system with the Lagrangian action given by

$$
\mathscr{L}_{g}(q)=\int_{0}^{1} \frac{1}{2} q_{t}^{2}+\frac{1}{2} q^{2}-g(q, t) d t .
$$

The braid class $[q]$ rel $Q$ is bounded due to the special strands \pm 1 and all free strands $q$ satisfy $-1 \leq q(t) \leq 1$. Therefore, the set of critical points $q_{0}$ of $\mathscr{L}_{g}$ for which $q_{0}$ rel $Q$ lies in $[q] \operatorname{rel} Q$ is a compact set and is denoted by $\operatorname{Crit} \mathscr{L}_{g}([q] \operatorname{rel} Q)$.

Proposition 5 Let $[x$ rel $y]$ be a proper relative braid class and let $[q$ rel $Q]$ be the class of induced (Legendrian) braid diagrams. Suppose all critical points in $\mathrm{Crit}_{\mathscr{L}_{\mathrm{g}}}([q] \mathrm{rel} Q)$ are non-degenerate, then

$$
\sum_{q_{0} \in \operatorname{Crit} \mathscr{L}_{g}([q] \operatorname{rel} Q)}(-1)^{\gamma\left(q_{0}\right)}=\chi(x \text { rel } y) .
$$

Proof The critical points in Crit $\mathscr{L}_{g}([q] \mathrm{rel} Q)$ are in one-to-one correspondence with the zeroes of the equation

$$
\Phi_{H_{L}}(x)=x-\mathcal{N}_{\mu}^{-1}\left(\nabla H_{L}(x, t)+\mu x\right)=0,
$$

on the set $\Omega_{\mathbb{R}^{2}} \subset H^{1}(\mathbb{R} / \mathbb{Z})$, which is defined as the set of functions $x \in H^{1}(\mathbb{R} / \mathbb{Z})$ such that $x$ rel $y_{L}$ lies in $\left[x_{L}\right]_{\mathbb{R}^{2}}$ rel $y_{L}$. From Lemma 8 we have that the zeroes of $\Phi_{H_{L}}$ satisfy $\left|x_{0}(t)\right| \leq R^{\prime}$, with $R^{\prime}>1$, and thus $\Phi_{H_{L}}^{-1}(0) \cap \Omega_{\mathbb{R}^{2}} \subset B_{R^{\prime \prime}}(0) \subset H^{1}(\mathbb{R} / \mathbb{Z})$ for some $R^{\prime \prime}>0$ which implies that $\Phi_{H_{L}}$ is a proper mapping on $\Omega_{\mathbb{R}^{2}}$ and $\operatorname{deg}_{L S}\left(\Phi_{H_{L}}, \Omega_{\mathbb{R}^{2}}, 0\right)$ is well-defined. As before we may assume that $H_{L}$ is a generic Hamiltonian and by (19) we 
have

$$
\begin{aligned}
\operatorname{deg}_{L S}\left(\Phi_{H_{L}}, \Omega_{\mathbb{R}^{2}}, 0\right) & =\sum_{x_{0} \in \Phi_{H_{L}}^{-1}(0) \cap \Omega_{\mathbb{R}^{2}}} \operatorname{deg}_{L S}\left(D_{x} \Phi_{H_{L}}\left(x_{0}\right)\right) \\
=- & \sum_{x_{0} \in \Phi_{H_{L}}^{-1}(0) \cap \Omega_{\mathbb{R}^{2}}}(-1)^{\mu}{ }^{C Z}\left(x_{0}\right)=-\sum_{q_{0} \in \operatorname{Crit} \mathscr{L}_{g}([q] \operatorname{rel} Q)}(-1)^{\gamma\left(q_{0}\right)},
\end{aligned}
$$

where the latter equality follows from Lemma 11.

We are now in a position to use a homotopy argument. We can scale the skeleton $y$ to a braid $\rho y$ such that the induced rescaled Legendrian braid $\rho y_{L}$ is supported in $\mathbb{D}^{2}$. By Lemma 7, $y$ is isotopic to $y_{L}$ and scaling defines an isotopy between $y_{L}$ and $\rho y_{L}$. Denote the composite isotopy from $y$ to $\rho y_{L}$ by $y_{\alpha}$. For a Hamiltonian $H \in \mathscr{H}(y)$, Eq. (23) implies that $\operatorname{deg}_{L S}\left(\Phi_{H}, \Omega, 0\right)=-\chi(x$ rel $y)$ for the fiber $[x]$ rel $y$. Since the latter is fiber independent we also have $\operatorname{deg}_{L S}\left(\Phi_{H_{\rho}}, \Omega_{\rho}, 0\right)=-\chi(x$ rel $y)$, where $\Omega_{\rho} \subset H^{1}(\mathbb{R} / \mathbb{Z})$ is the set of functions $x_{0} \in H^{1}(\mathbb{R} / \mathbb{Z})$ such that $x_{0}$ rel $\rho y_{L}$ lies in $\left[\rho x_{L}\right]$ rel $\rho y_{L} \subset[x$ rel $y]$ and $H_{\rho} \in \mathscr{H}\left(\rho y_{L}\right)$. Now extend $H_{\rho}$ to $\mathbb{R}^{2} \times \mathbb{R} / \mathbb{Z}$, such that Hypotheses (h1)-(h3) are satisfied for some $R>1$. We denote the Hamiltonian again by $H_{\rho}$. By construction all zeroes of $\Phi_{H_{\rho}}$ in $\Omega_{\rho}$ are supported in $\mathbb{D}^{2}$. Define $\Omega_{\rho, \mathbb{R}^{2}}$ as the set of functions $x_{0} \in H^{1}(\mathbb{R} / \mathbb{Z})$ such that $x_{0}$ rel $\rho y_{L}$ lies in $\left[\rho x_{L}\right]_{\mathbb{R}^{2}}$ rel $\rho y_{L}$. By the properness of $[x$ rel $y]$ the zeroes of $\Phi_{H_{\rho}}$ in $\left[\rho x_{L}\right]_{\mathbb{R}^{2}}$ rel $\rho y_{L}$ are also supported in $\mathbb{D}^{2}$. Indeed, since $\partial \mathbb{D}^{2}$ is invariant for the Hamiltonian vector field, a zero takes values either inside or outside $\mathbb{D}^{2}$. Combining the latter with the properness of the braid class implies that a zero takes values strictly inside $\mathbb{D}^{2}$ and therefore $\operatorname{deg}_{L S}\left(\Phi_{H_{\rho}}, \Omega_{\rho, \mathbb{R}^{2}}, 0\right)=$ $\operatorname{deg}_{L S}\left(\Phi_{H_{\rho}}, \Omega_{\rho}, 0\right)=-\chi(x$ rel $y)$.

For the next homotopy we keep the skeleton $\rho y_{L}$ fixed as well as the domain $\Omega_{\rho, \mathbb{R}^{2}}$. Consider the linear homotopy of Hamiltonians

$$
H_{1}(x, t ; \alpha)=\frac{1}{2} p^{2}-\frac{1}{2} q^{2}+(1-\alpha) h_{\rho}(x, t)+\alpha g_{\rho}(q, t),
$$

where $H_{\rho, L}(t, x)=\frac{1}{2} p^{2}-\frac{1}{2} q^{2}+g_{\rho}(q, t)$ given by Lemma 10 . This defines an admissible homotopy since $\rho y_{L}$ is a skeleton for all $\alpha \in[0,1]$. The uniform estimates are obtained by Lemma 9, which, by the homotopy invariance of the Leray-Schauder degree yields: $\operatorname{deg}_{L S}\left(\Phi_{H_{\rho, L}}, \Omega_{\rho, \mathbb{R}^{2}}, 0\right)=\operatorname{deg}_{L S}\left(\Phi_{H_{\rho}}, \Omega_{\rho, \mathbb{R}^{2}}, 0\right)=-\chi(x$ rel $y)$. Finally, we scale $\rho y_{L}$ to $y_{L}$ via $y_{\alpha, L}=(1-\alpha) \rho y_{L}+\alpha y_{L}$. We consider the homotopy

$$
H_{2}(x, t ; \alpha)=\frac{1}{2} p^{2}-\frac{1}{2} q^{2}+g(q, t ; \alpha),
$$

between $H_{L}$ and $H_{\rho, L}$, where $g(q, t ; \alpha)$ is obtained by applying Lemma 10 to $y_{\alpha, L}$. From the homotopy invariance of the Leray-Schauder degree we obtain

$$
\operatorname{deg}_{L S}\left(\Phi_{H_{L}}, \Omega_{\mathbb{R}^{2}}, 0\right)=\operatorname{deg}_{L S}\left(\Phi_{H_{\rho, L}}, \Omega_{\rho, \mathbb{R}^{2}}, 0\right)=-\chi(x \text { rel } y) .
$$

Combining the latter with Eq. (35) completes the proof.

\section{The proof of Theorem 2}

The Lagrangian formulation of the Poincaré-Hopf index formula in Proposition 5 allows for another simplification via a finite dimensional reduction. 


\subsection{Discretized braid classes}

The critical point theory for the Lagrangians in (33) can be treated using a variation on the method of broken geodesics. If $1 / d>0$ is chosen sufficiently small, then the integral

$$
S_{i}\left(q_{i}, q_{i+1}\right)=\min _{\substack{q(t) \in E_{i}\left(q_{i}, q_{i+1}\right) \\|q(t)| \leq 1}} \int_{\tau_{i}}^{\tau_{i+1}} \frac{1}{2} q_{t}^{2}+\frac{1}{2} q^{2}-g(q, t) d t,
$$

has a unique minimizer $q^{i}$, where $E_{i}\left(q_{i}, q_{i+1}\right)=\left\{q \in H^{1}\left(\tau_{i}, \tau_{i+1}\right) \mid q\left(\tau_{i}\right)=q_{i}, q\left(\tau_{i+1}\right)=\right.$ $\left.q_{i+1}\right\}$, and $\tau_{i}=i / d$. Moreover, if $1 / d$ is small, then the minimizers are non-degenerate and $S_{i}$ is a smooth function of $q_{i}$ and $q_{i+1}$. Critical points $q$ of $\mathscr{L}_{g}$ with $|q(t)| \leq 1$ correspond to sequences $q_{D}=\left(q_{0}, \ldots, q_{d}\right)$, with $q_{0}=q_{d}$, which are critical points of the discrete action

$$
\mathscr{W}\left(q_{D}\right)=\sum_{i=0}^{d-1} S_{i}\left(q_{i}, q_{i+1}\right)
$$

A concatenation $\#_{i} q^{i}$ of minimizers $q^{i}$ is continuous and is an element in the function space $H^{1}(\mathbb{R} / \mathbb{Z})$. Such a function is referred to as a broken geodesic. The set of broken geodesics $\#_{i} q^{i}$ is denoted by $E\left(q_{D}\right)$ and standard arguments using the non-degeneracy of minimizers $q^{i}$ show that $E\left(q_{D}\right) \hookrightarrow H^{1}(\mathbb{R} / \mathbb{Z})$ is a smooth, $d$-dimensional submanifold in $H^{1}(\mathbb{R} / \mathbb{Z})$. The submanifold $E\left(q_{D}\right)$ is parametrized by sequences in $D_{d}=\left\{q_{D} \in \mathbb{R}^{d}|| q_{i} \mid \leq 1\right\}$ and yields the commuting diagram:

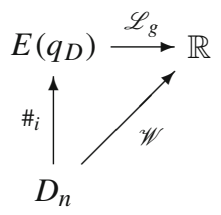

cf. [11]. In the above diagram $\#_{i}$ is regarded as a mapping $q_{D} \mapsto \#_{i} q^{i}$, where the minimizers $q_{i}$ are determined by $q_{D}$. The tangent space to $E\left(q_{D}\right)$ at a broken geodesic $\#_{i} q^{i}$ is identified by

$$
\begin{gathered}
T_{\#_{i} q^{i}} E\left(q_{D}\right)=\left\{\psi \in H^{1}(\mathbb{R} / \mathbb{Z}) \mid-\psi_{t t}+\psi-g_{q q}\left(q^{i}(t), t\right) \psi=0,\right. \\
\left.\psi\left(\tau_{i}\right)=\delta q_{i}, \quad \psi\left(\tau_{i+1}\right)=\delta q_{i+1}, \quad \delta q_{i} \in \mathbb{R}, \forall i\right\},
\end{gathered}
$$

and $\#_{i} q^{i}+T_{\#_{i} q^{i}} E\left(q_{D}\right)$ is the tangent hyperplane at $\#_{i} q^{i}$. For $H^{1}(\mathbb{R} / \mathbb{Z})$ we have the following decomposition for any broken geodesic $\#_{i} q^{i} \in E\left(q_{D}\right)$ :

$$
H^{1}(\mathbb{R} / \mathbb{Z})=E^{\prime} \oplus T_{\#_{i} q^{i}} E\left(q_{D}\right),
$$

where $E^{\prime}=\left\{\eta \in H^{1}(\mathbb{R} / \mathbb{Z}) \mid \eta\left(\tau_{i}\right)=0, \quad \forall i\right\}$. To be more specific the decomposition is orthogonal with respect to the quadratic form

$$
D_{q}^{2} \mathscr{L}_{g}(q) \phi \widetilde{\phi}=\int_{0}^{1} \phi_{t} \widetilde{\phi}_{t}+\phi \widetilde{\phi}-g_{q q}(q(t), t) \phi \widetilde{\phi} d t, \quad \phi, \widetilde{\phi} \in H^{1}(\mathbb{R} / \mathbb{Z})
$$


Indeed, let $\eta \in E^{\prime}$ and $\psi \in T_{\#_{i} q^{i}} E\left(q_{D}\right)$, then

$$
\begin{aligned}
D_{q}^{2} \mathscr{L}_{g}\left(\#_{i} q^{i}\right) \eta \psi & =\sum_{i} \int_{\tau_{i}}^{\tau_{i+1}} \eta_{t} \psi_{t}+\eta \psi-g_{q q}\left(q^{i}(t), t\right) \xi \eta d t \\
& =\left.\sum_{i} \psi_{t} \eta\right|_{\tau_{i}} ^{\tau_{i+1}}-\sum_{i} \int_{\tau_{i}}^{\tau_{i+1}}\left[-\psi_{t t}+\psi+g_{q q}\left(q^{i}(t), t\right) \psi\right] \eta d t=0 .
\end{aligned}
$$

Let $\phi=\eta+\psi$, then

$$
D_{q}^{2} \mathscr{L}_{g}\left(\#_{i} q^{i}\right) \phi \widetilde{\phi}=D_{q}^{2} \mathscr{L}_{g}\left(\#_{i} q^{i}\right) \eta \tilde{\eta}+D_{q}^{2} \mathscr{L}_{g}\left(\#_{i} q^{i}\right) \psi \tilde{\psi}
$$

by the above orthogonality. By construction the minimizers $q^{i}$ are non-degenerate and therefore $\left.D_{q}^{2} \mathscr{L}_{g}\right|_{E^{\prime}}$ is positive definite. This implies that the Morse index of a (stationary) broken geodesic is determined by $\left.D_{q}^{2} \mathscr{L}_{g}\right|_{T_{\#_{i}} q^{i}} E\left(q_{D}\right)$. By the commuting diagram for $\mathscr{W}$ this implies that the Morse index is given by the quadratic form $D_{q_{D}}^{2} \mathscr{W}\left(q_{D}\right)$. We have now proved the following lemma that relates the Morse index of critical points of the discrete action $\mathscr{W}$ to Morse index of the 'full' action $\mathscr{L}_{g}$.

Lemma 12 Let $q$ be a non-degenerate critical point of $\mathscr{L}_{g}$ and $q_{D}$ the corresponding critical point of $\mathscr{W}$, then the Morse indices are the same i.e. $\gamma(q)=\gamma\left(q_{D}\right)$.

For a 1-periodic function $q(t)$ we define the mapping

$$
q \stackrel{D_{d}}{\longrightarrow} q_{D}=\left(q_{0}, \ldots, q_{d}\right), \quad q_{i}=q(i / d), \quad i=0, \ldots, d,
$$

and $q_{D}$ is called the discretization of $q$. The linear interpolation

$$
q_{D} \mapsto \ell_{q_{D}}(t)=\#_{i}\left[q_{i}+\frac{q_{i+1}-q_{i}}{d} t\right],
$$

reconstructs a piecewise linear 1-periodic function. For a relative braid diagram $q$ rel $Q$, let $q_{D}$ rel $Q_{D}$ be its discretization, where $Q_{D}$ is obtained by applying $D_{d}$ to every strand in $Q$. A discretization $q_{D}$ rel $Q_{D}$ is admissible if $\ell_{q_{D}}$ rel $\ell_{Q_{D}}$ is homotopic to $q$ rel $Q$, i.e. $\ell_{q_{D}} \operatorname{rel} \ell_{Q_{D}} \in[q \operatorname{rel} Q]$. Define the discrete relative braid class $\left[q_{D} \operatorname{rel} Q_{D}\right]$ as the set of 'discrete relative braids' $q_{D}^{\prime}$ rel $Q_{D}^{\prime}$, such that $\ell_{q_{D}^{\prime}}$ rel $\ell_{Q_{D}^{\prime}} \in[q$ rel $Q]$. The associated fibers are denoted by $\left[q_{D}\right]$ rel $Q_{D}$. It follows from [9, Prop. 27] that [ $q_{D}$ rel $\left.Q_{D}\right]$ is guaranteed to be connected when

$$
d>\#\{\text { crossings in } q \operatorname{rel} Q\},
$$

i.e. for any two discrete relative braids $q_{D}$ rel $Q_{D}$ and $q_{D}^{\prime}$ rel $Q_{D}^{\prime}$, there exists a homotopy $q_{D}^{\alpha}$ rel $Q_{D}^{\alpha}$ (discrete homotopy) such that $\ell_{q_{D}^{\alpha}}$ rel $\ell_{Q_{D}^{\alpha}}$ is a path in $[q$ rel $Q]$. Note that fibers are not necessarily connected!

We showed above that if $1 / d>0$ is sufficiently small, then the critical points of $\mathscr{L}_{g}$, with $|q| \leq 1$, are in one-to-one correspondence with the critical points of $\mathscr{W}$, and, if nondegenerate, their Morse indices coincide by Lemma 12. Moreover, if $1 / d>0$ is small enough, then for all critical points in $\operatorname{Crit}_{\mathscr{L}_{g}}([q] \mathrm{rel} Q)$, the associated discretizations are admissible and $\left[q_{D}\right.$ rel $\left.Q_{D}\right]$ is a connected set. The discretizations of the critical points in Crit $\mathscr{L}_{g}([q] \mathrm{rel} Q)$ are critical points $q_{D, 0}$ of $\mathscr{W}$ such that $q_{D, 0}$ rel $Q_{D}$ lies in the fiber $\left[q_{D}\right]$ rel $Q_{D}$ which are denoted by Crit $\mathscr{W}\left(\left[q_{D}\right]\right.$ rel $\left.Q_{D}\right)$. 
Proposition 6 Let $[x$ rel $y]$ be a proper relative braid class and let $\left[q_{D}\right.$ rel $\left.Q_{D}\right]$ be the class of addmissible discretized braids. Suppose the critical points in Crit $\mathscr{W}\left(\left[q_{D}\right] \mathrm{rel} Q_{D}\right)$ are non-degenerate, then

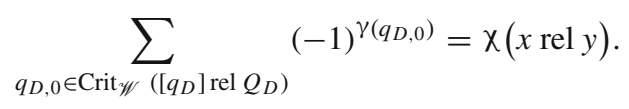

Proof Combine Proposition 5 and Lemma 12.

\subsection{The Conley index for discrete braids}

In [9] an invariant for discrete braid classes $\left[q_{D}\right.$ rel $\left.Q_{D}\right]$ is defined based on the Conley index. Recall from Sect. 1.3 that the invariant is given by

$$
\mathrm{HC}_{*}\left(\left[q_{D}\right] \operatorname{rel} Q_{D}\right)=\bigoplus_{j} H_{*}\left(N_{j}, N_{j}^{-}\right),
$$

and is well-defined for any $d>0$ for which there exist admissible discretizations. The invariant is independent of both the fiber and the discretization size $d$. For discretized action $\mathscr{W}$ which is a Morse function and for which $Q_{D}$ is a critical braid we have the relation:

$$
\sum_{q_{D, 0} \in \operatorname{Crit} \mathscr{W}\left(\left[q_{D}\right] \operatorname{rel} Q_{D}\right)}(-1)^{\gamma\left(q_{D, 0}\right)}=\chi\left(\mathrm{HC}_{*}\left(\left[q_{D}\right] \operatorname{rel} Q_{D}\right)\right)=: \chi\left(q_{D} \operatorname{rel} Q_{D}\right),
$$

cf. [9]. The latter can be computed for any admissible discretization and is an invariant for [ $q$ rel $Q$ ]. Combining Proposition 6 and Eq. (40) yields

$$
\chi(x \operatorname{rel} y)=\chi\left(q_{D} \operatorname{rel} Q_{D}\right)
$$

which completes the proof of Theorem 2 .

Remark 4 In this section we assumed without loss of generality that $x$ rel $y$ is augmented. Therefore, if $[x$ rel $y]$ is an arbitrary proper braid class, then

$$
\chi(x \operatorname{rel} y)=\chi\left(x_{L} \operatorname{rel} y_{L}^{*}\right)=\chi\left(q_{D} \operatorname{rel} Q_{D}^{*}\right) .
$$

\section{Examples}

We will illustrate by means of two examples that the Euler-Floer characteristic is computable and can be used to find 1-periodic closed integral curves of vector fields on the 2-disc.

\subsection{Example}

Figure 4 (left) shows the braid diagram $q$ rel $Q$ of a positive relative braid $x$ rel $y$. The discretization with $q_{D}$ rel $Q_{D}$, with $d=2$, is shown in Fig. 4 (right). The chosen discretization is admissible and defines the relative braid class $\left[q_{D}\right.$ rel $\left.Q_{D}\right]$. There are five strands, one is free and four are fixed. We denote the points on the free strand by $q_{D}=\left(q_{0}, q_{1}\right)$ and on the skeleton by $Q_{D}=\left\{Q^{1}, \ldots, Q^{4}\right\}$, with $Q^{i}=\left(Q_{0}^{i}, Q_{1}^{i}\right), i=1, \ldots, 4$.

In Fig. 5 (left) the relative braid class fiber $\left[q_{D}\right]$ rel $Q_{D}$ is depicted. The coordinate $q_{0}$ is allowed to move between $Q_{0}^{3}$ and $Q_{0}^{2}$ and $q_{1}$ remains in the same braid class if it varies between $Q_{1}^{1}$ and $Q_{1}^{4}$. For the values $q_{0}=Q_{0}^{3}$ and $q_{0}=Q_{0}^{2}$ the relative braid becomes singular and if $q_{0}$ crosses any of these values two intersections are created. If $q_{1}$ crosses the 

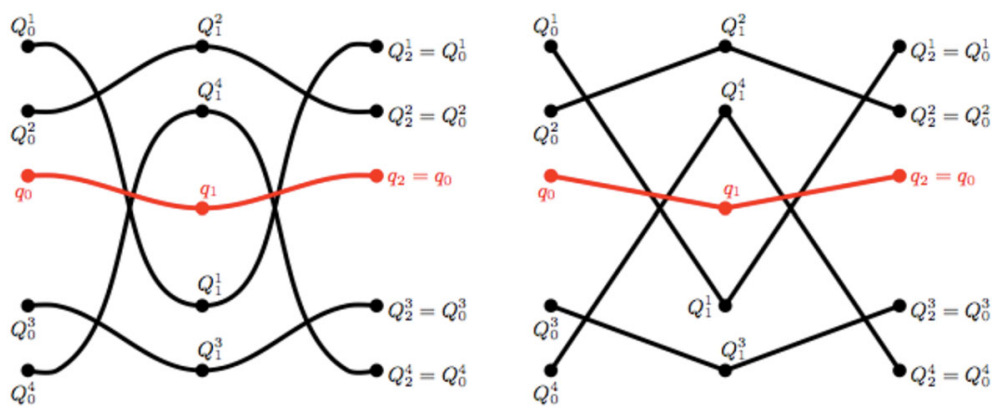

Fig. 4 A positive braid diagram (left) and an admissible discretization (right)
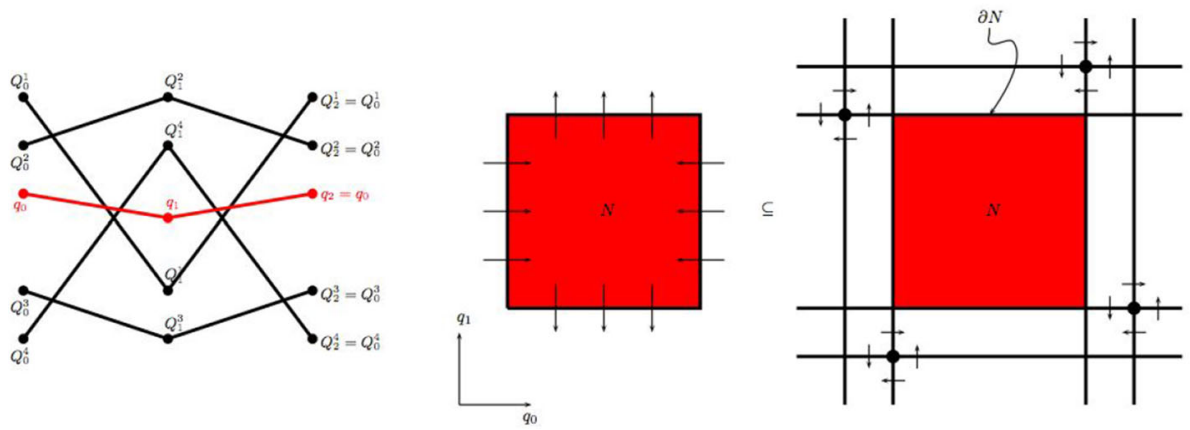

Fig. 5 The relative braid fiber $\left[q_{D}\right] \operatorname{rel} Q_{D}$ and $N=\operatorname{cl}\left(\left[q_{D}\right] \operatorname{rel} Q_{D}\right)$

values $Q_{1}^{1}$ or $Q_{1}^{4}$ two intersections are destroyed. This provides the desired co-orientation, see Fig. 5 (middle). The relative braid class fiber $\left[q_{D}\right]$ rel $Q_{D}$ consists of one component and

$$
N=\operatorname{cl}\left(\left[q_{D} \operatorname{rel} Q_{D}\right]\right)=\left\{\left(q_{0}, q_{1}\right): Q_{0}^{3} \leq q_{0} \leq Q_{0}^{2}, Q_{1}^{1} \leq q_{1} \leq Q_{1}^{4}\right\} .
$$

The exit set is given by

$$
N^{-}=\left\{\left(q_{0}, q_{1}\right): q_{1}=Q_{1}^{1}, \text { or } q_{1}=Q_{1}^{4}\right\} .
$$

For the Conley index we obtain:

$$
\mathrm{HC}_{k}\left(\left[q_{D}\right] \operatorname{rel} Q_{D}\right)=H_{k}\left(N, N^{-} ; \mathbb{Z}\right) \cong \begin{cases}\mathbb{Z} & k=1 \\ 0 & \text { otherwise }\end{cases}
$$

The Euler characteristic of $\chi\left(\mathrm{HC}_{*}\left(\left[q_{D}\right]\right.\right.$ rel $\left.\left.Q_{D}\right)\right)$ is denoted by $\chi\left(q_{D}\right.$ rel $\left.Q_{D}\right)$, cf. (40). The Euler-Floer characteristic of $x$ rel $y$ can be compute via Theorem 2:

$$
\chi(x \operatorname{rel} y)=\chi\left(q_{D} \operatorname{rel} Q_{D}^{*}\right)=\chi\left(q_{D} \operatorname{rel} Q_{D}\right)=-1 \neq 0
$$

From Corollary 1 we conclude that any vector field for which $y$ is a skeleton has at least one closed integral curve $x_{0}$ such that $x_{0}$ rel $y \in[x]$ rel $y$.

\subsection{Example}

The theory in this paper can also be used to find additional closed integral curves by concatenating the skeleton $y$. As in the previous example $y$ is given by Fig. 4. Glue $\ell$ copies 


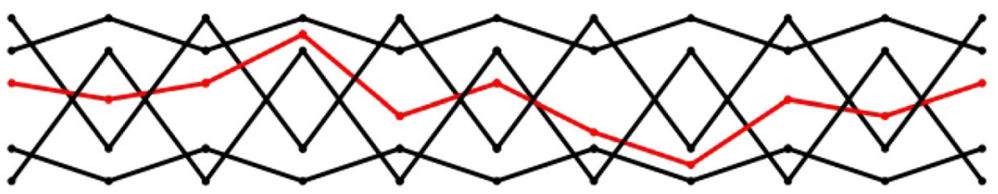

Fig. 6 A discretization of a braid class with a fivefold concatenation of the skeleton $y$. The number of odd anchor points in middle position is $\mu=3$

of the skeleton $y$ to its $\ell$-fold concatenation and reparametrize time by $t \mapsto \ell \cdot t$. Denote the rescaled $\ell$-fold concatenation of $y$ by $\#_{\ell} y$. Choose $d=2 \ell$ and discretize $\#_{\ell} y$ as in the previous example. Figure 6 shows a discretized admissible representative $q_{D}$ rel $\#_{\ell} Q_{D}$ of a proper relative braid class $\left[x\right.$ rel $\left.\#_{\ell} y\right]$. For the skeleton $\#_{\ell} Q_{D}$ we can construct $3^{\ell}-2$ proper relative braid classes in the following way: the even anchor points of the free strand $q_{D}$ are always in the middle and for the odd anchor points we have 3 possible choices: bottom, middle, top ( 2 braids are not proper). The Conley index of the $3^{\ell}-2$ different proper discrete relative braid classes can be computed as follows.

The configuration space $N=\operatorname{cl}\left(\left[q_{D}\right] \mathrm{rel} \#_{\ell} Q_{D}\right)$ is given by a cartesian product of $2 \ell$ closed intervals, and therefore a $2 \ell$-dimensional hypercube. As in the previous example the co-orientation on the boundary of the exit set $N^{-}$is found as a union of faces with an outward pointing co-orientation. Due to the product structure of $N$, the set $N^{-}$is determined by the odd anchor points in the middle position. Denote the number of middle positions at odd anchor points by $\mu$. In this way $N^{-}$consists of opposite faces at at odd anchor points in middle position, see Fig. 6. Therefore

$$
\operatorname{HC}_{k}\left(\left[q_{D}\right] \operatorname{rel} \#_{\ell} Q_{D}\right)=H_{k}\left(N, N^{-}\right)= \begin{cases}\mathbb{Z}_{2} & k=\mu \\ 0 & k \neq \mu,\end{cases}
$$

and the Euler-Floer characteristic is given by

$$
\chi\left(x \operatorname{rel} \#_{\ell} y\right)=(-1)^{\mu} \neq 0 .
$$

Let $X(x, t)$ be a vector field for which $y$ is a skeleton of closed integral curves, then $\#_{\ell} y$ is a skeleton for the vector field $X^{\ell}(x, t):=\ell X(x, \ell t)$. From Corollary 1 we derive that there exists a closed integral curve in each of the $3^{\ell}-2$ proper relative classes $[x]$ rel $y$ described above. For the original vector field $X$ this yields $3^{\ell}-2$ distinct closed integral curves in the complement of $y$. Using the arguments in [16] we obtain a compact invariant set with positive topological entropy for any vector field $X$ for which $y$ is a skeleton of integral curves.

Acknowledgements The authors wish to thank J.B. van den Berg for the many stimulating discussions on the subject of Braid Floer Homology. We particularly thank the referee for carefully reading the manuscript and suggesting the many improvements concerning both exposition and content of the paper.

Open Access This article is distributed under the terms of the Creative Commons Attribution 4.0 International License (http://creativecommons.org/licenses/by/4.0/), which permits unrestricted use, distribution, and reproduction in any medium, provided you give appropriate credit to the original author(s) and the source, provide a link to the Creative Commons license, and indicate if changes were made.

\section{References}

1. Abbondandolo, A.: On the Morse index of Lagrangian systems. Nonlinear Anal. 53(3-4), 551-566 (2003)

2. Duistermaat, J.J.: On the Morse index in variational calculus. Adv. Math. 21(2), 173-195 (1976) 
3. Fitzpatrick, P.M., Pejsachowicz, Jacobo: An extension of the Leray-Schauder degree for fully nonlinear elliptic problems, Nonlinear functional analysis and its applications, Part 1 (Berkeley, Calif., 1983), In: Proceedings of the Symposium Pure Mathematics vol. 45, Amer. Math. Soc., Providence, RI, 1986, pp. 425-438. MR 843576

4. Fitzpatrick, P.M., Pejsachowicz, J.: The fundamental group of the space of linear Fredholm operators and the global analysis of semilinear equations, Fixed point theory and its applications (Berkeley, CA, 1986), Contemp. Math., vol. 72, Amer. Math. Soc., Providence, RI, 1988, pp. 47-87. MR 956479 (89h:47097)

5. Fitzpatrick, P.M., Pejsachowicz, J.: Parity and generalized multiplicity. Trans. Amer. Math. Soc. 326(1), 281-305 (1991). MR 1030507 (91j:58038)

6. Fitzpatrick, P.M., Pejsachowicz, J., Rabier, P.: The degree of proper $c^{2}$-fredholm mappings. J. für Reine Angew. Math. 427, 1-33 (1992)

7. Fitzpatrick, P.M., Pejsachowicz, J., Recht, L.: Spectral flow and bifurcation of critical points of stronglyindefinite functionals. i. general theory. J. Funct. Anal. 162(1), 52-95 (1999)

8. Floer, A.: Symplectic fixed points and holomorphic spheres. Comm. Math. Phys. 120(4), 575-611 (1989). MR MR987770 (90e:58047)

9. Ghrist, R., van den Berg, J.B., Vandervorst, R.C.: Morse theory on spaces of braids and lagrangian dynamics. Invent. Math. 152(2), 369-432 (2003)

10. Lloyd, N.G.: Degree Theory. Cambridge University Press, Cambridge, (1978). Cambridge Tracts in Mathematics, No. 73. MR MR0493564 (58 \#12558)

11. Milnor, J.: Morse theory, Based on lecture notes by M. Spivak and R. Wells. Annals of Mathematics Studies, No. 51, Princeton University Press, Princeton, N.J., (1963)

12. Robbin, J., Salamon, D.: The Maslov index for paths. Topology 32(4), 827-844 (1993). MR MR1241874 (94i:58071)

13. Robbin, J., Salamon, D.: The spectral flow and the Maslov index. Bull. Lond. Math. Soc. 27, 1-33 (1995)

14. Salamon, D.: Lectures on Floer homology. Symplectic Geom. Topol. 7, 143-229 (1999)

15. Salamon, Dietmar, Zehnder, Eduard: Morse theory for periodic solutions of Hamiltonian systems and the Maslov index. Commun. Pure Appl. Math. 45(10), 1303-1360 (1992)

16. van den Berg, J.B., Vandervorst, R.C., Wójcik, W.: Chaos in orientation reversing twist maps on the plane. Topol. Appl. 154, 2580-2606 (2007)

17. van den Berg, J.B., Ghrist, R., Vandervorst, R.C., Wójcik, W.: Braid Floer homology. J. Differ. Equ. 259(5), 1663-1721 (2015). MR 3349416 